\title{
Predicting Human Thermal Comfort in Automobiles
}

\author{
John Rugh, Project Leader, Vehicle Ancillary Loads \\ Desikan Bharathan, Ph.D., Principal Engineer \\ Larry Chaney, Senior Engineer
}

June 13, 2005

Advanced Simulation Technologies User Meeting 2005

Graz, Austria

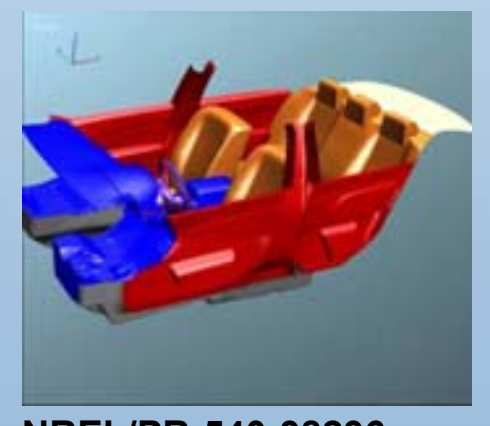

NREL/PR-540-38236

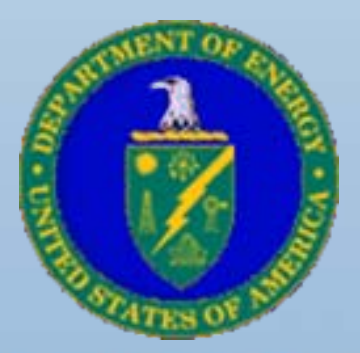

NREL is operated by Midwest Research Institute. Battelle

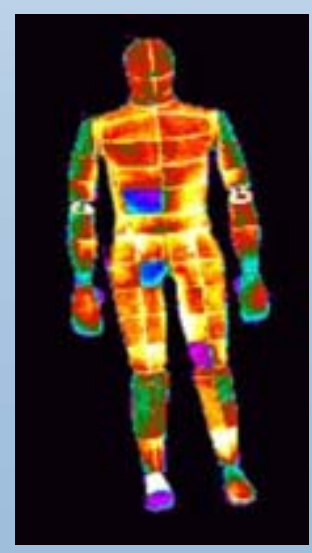




\section{Outline}

- Introduction

- Integrated Modeling Process for Cabin Thermal Management

- Vehicle Solar Load Estimator (VSOLE ${ }^{\circledR}$ )

- Thermal Comfort Tools

> Manikin

> Human Thermal Physiological Model

> Human Thermal Comfort Empirical Model

- Linking the Models

- Conclusions 


\section{Modeled U.S. Mobile AC Fuel Use}

7.0 billion gallons used for air conditioning annually

Equivalent to $5.5 \%$ of the national fuel use,

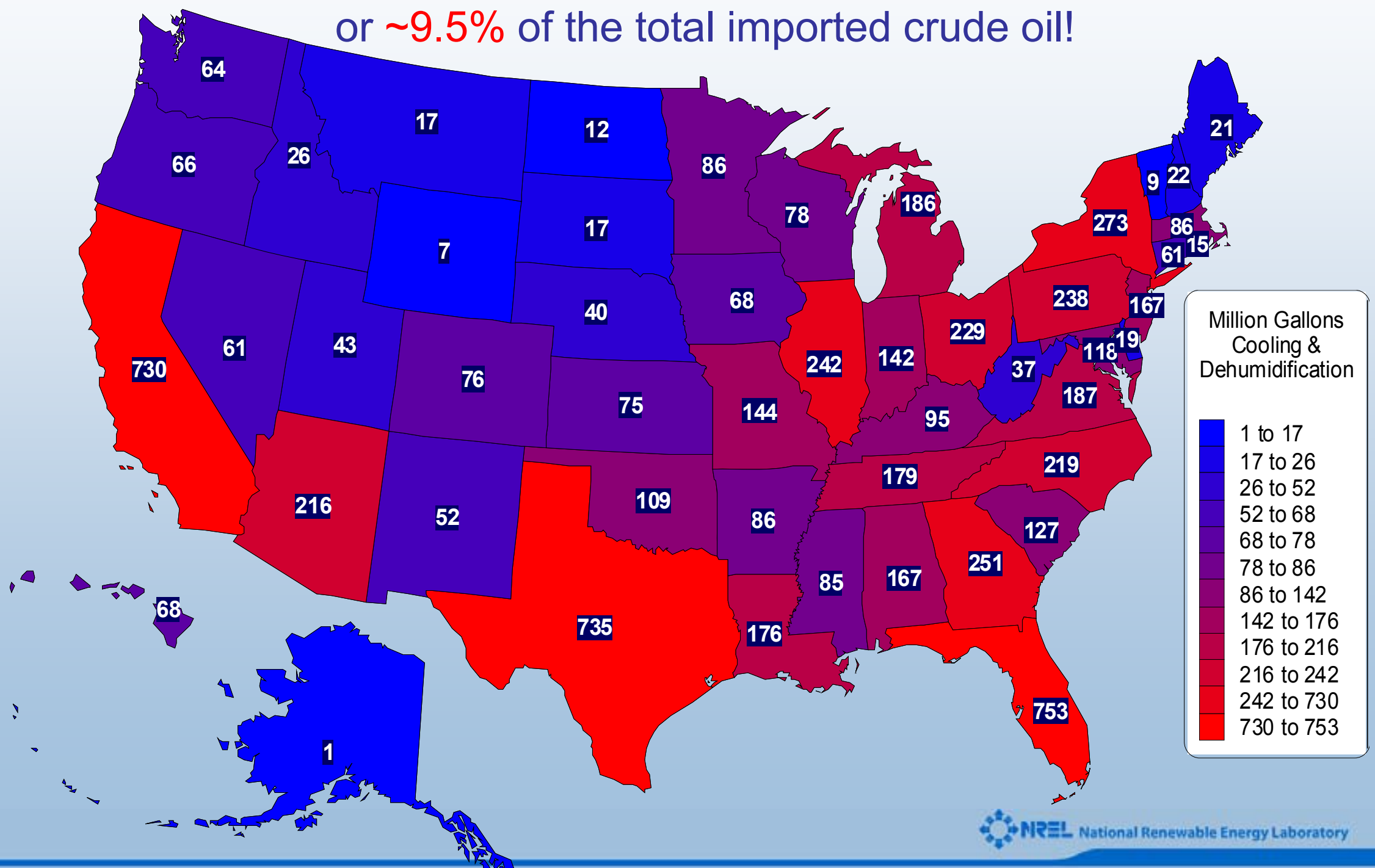




\section{Objectives}

- Increase national energy security by reducing fuel use for vehicle climate control systems

- Show/demonstrate technology that can reduce the fuel used by LD vehicles' ancillary systems

- Develop tools to evaluate the effectiveness of energy-efficient systems including:

- comfort, cost, practicality, ease-of-use, reliability ...
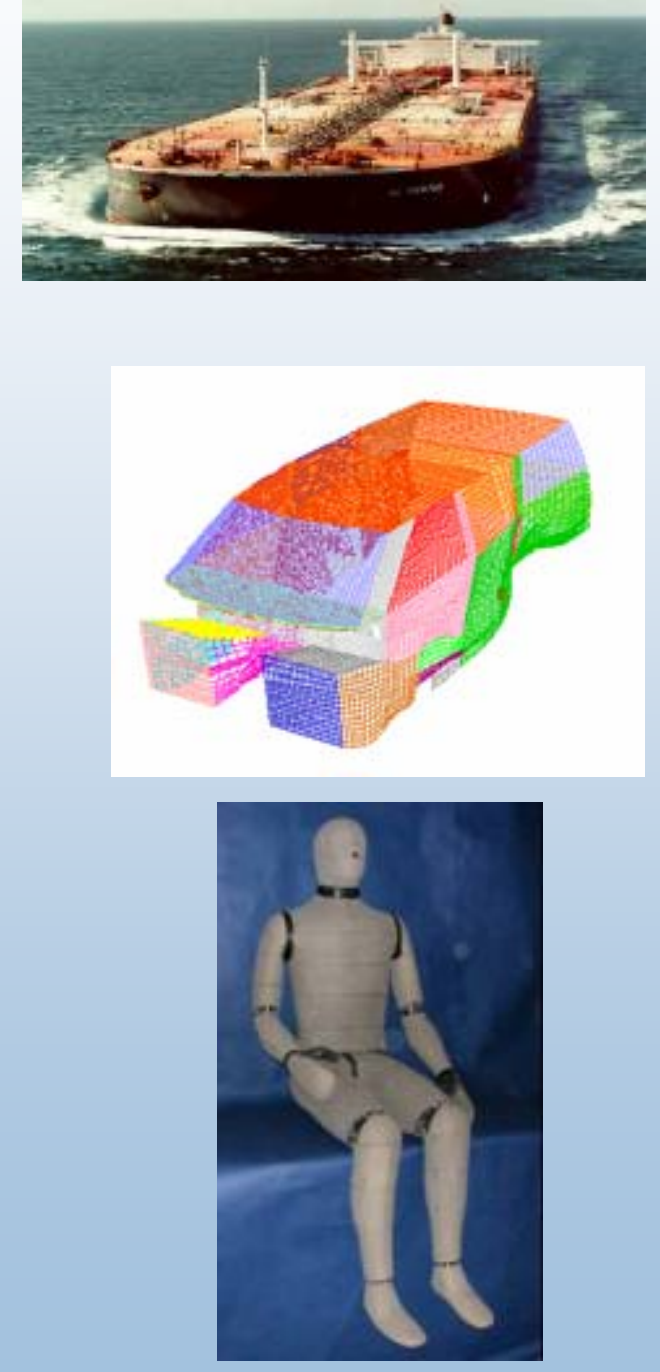


\section{Integrated Modeling}

Mesh the geometry of your vehicle

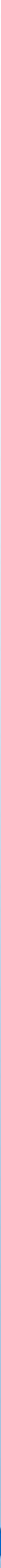




\section{Using ANSA Software for CAD Clean-Up}

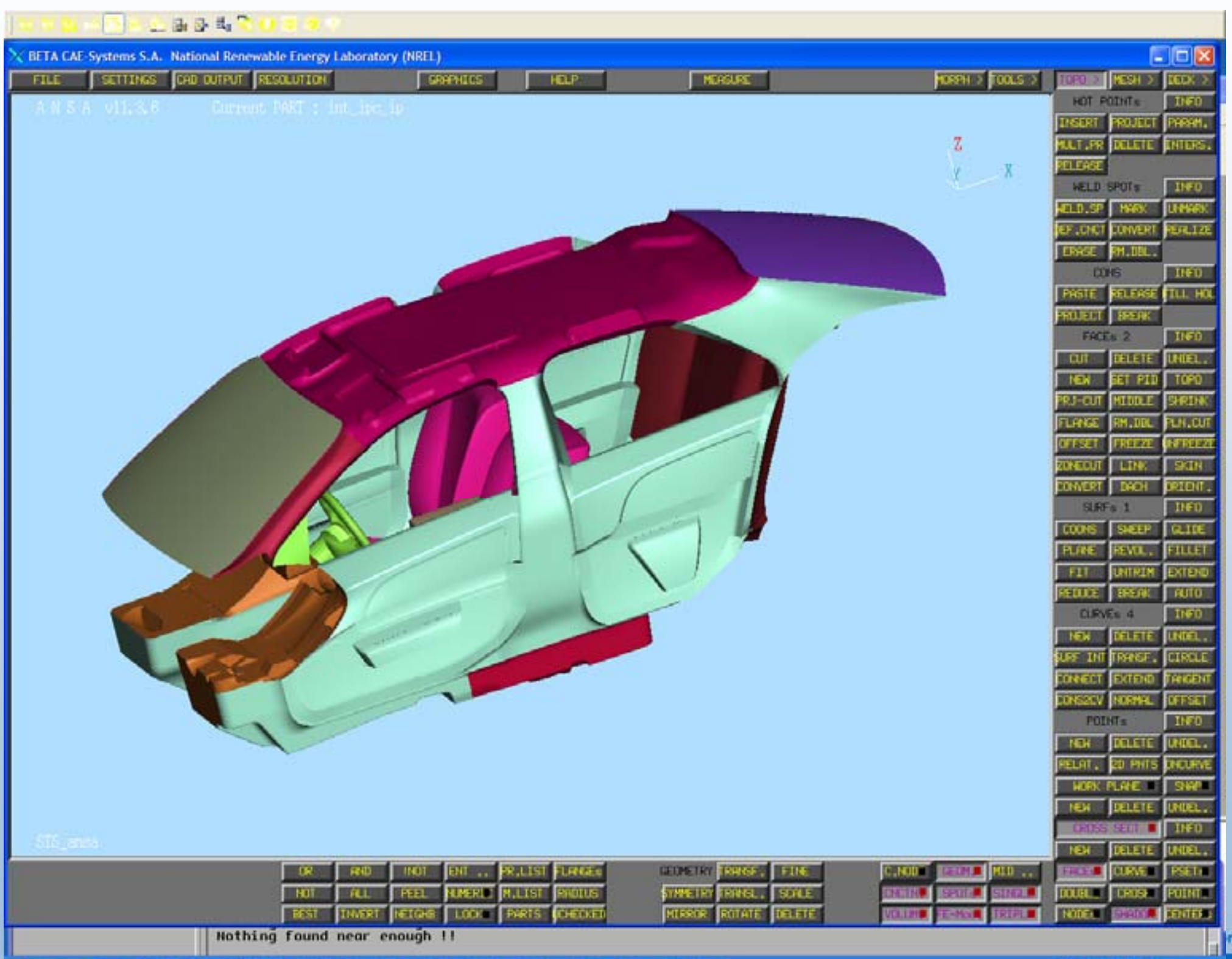

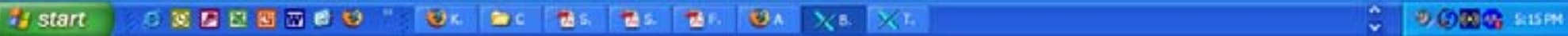




\section{Getting ADAM in the Drivers Seat}
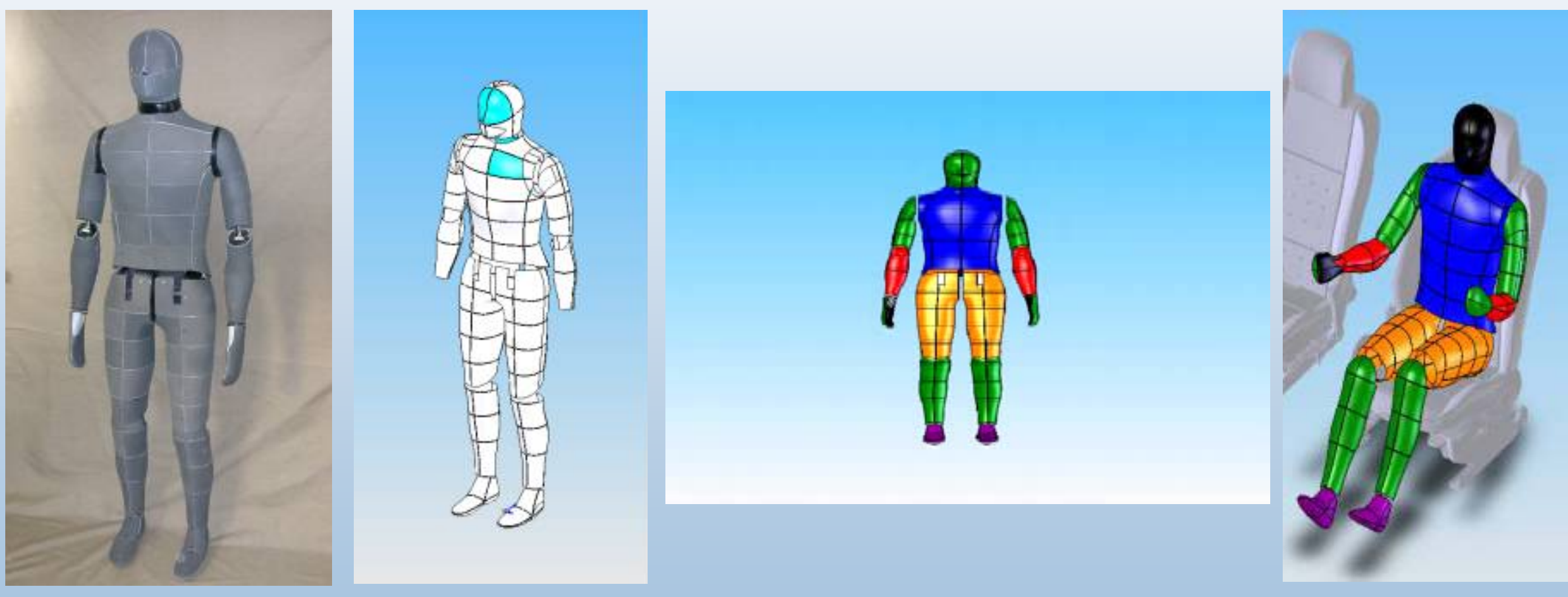


\section{Integrated Modeling}

Find the solar radiation in your city

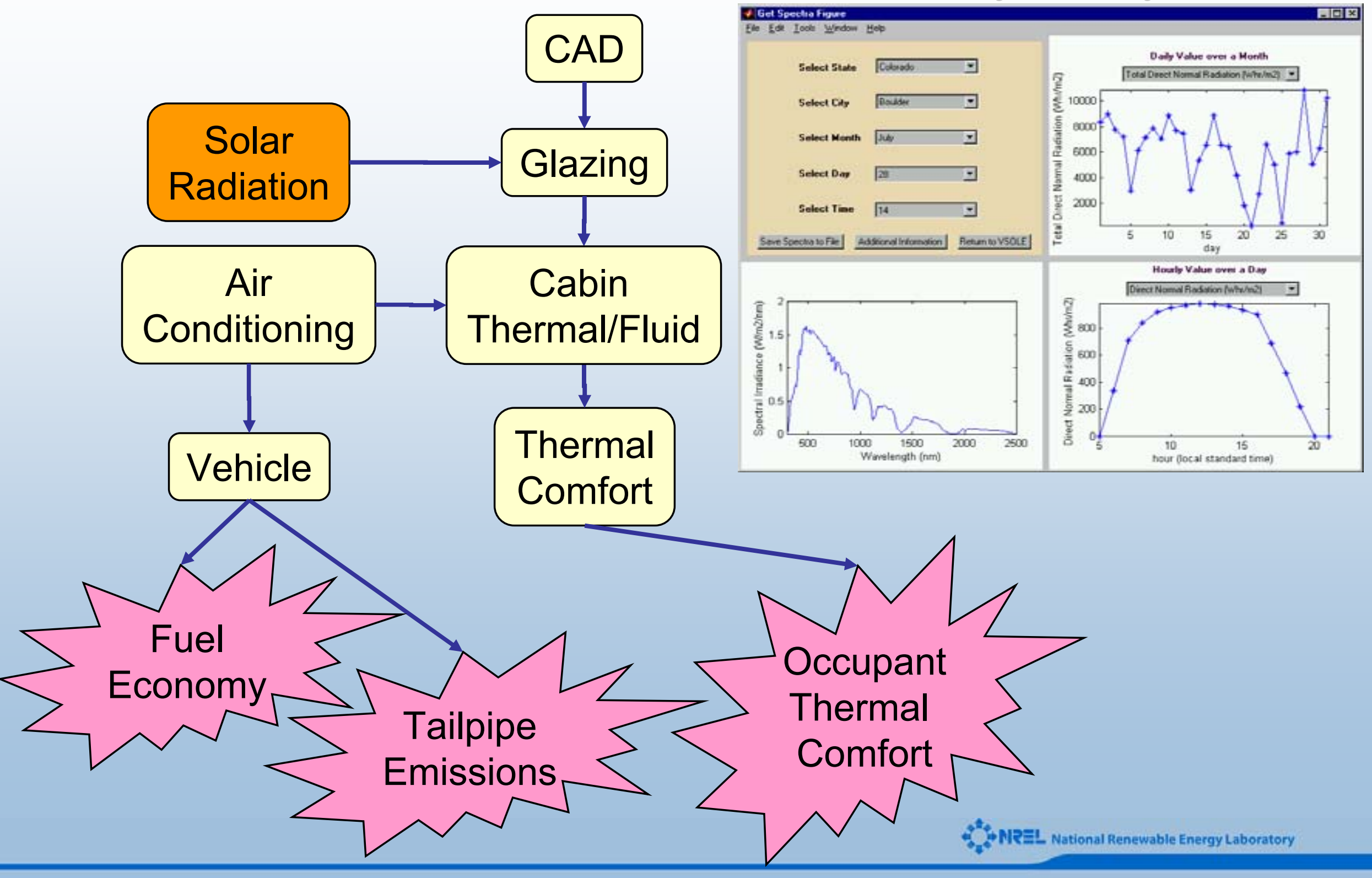




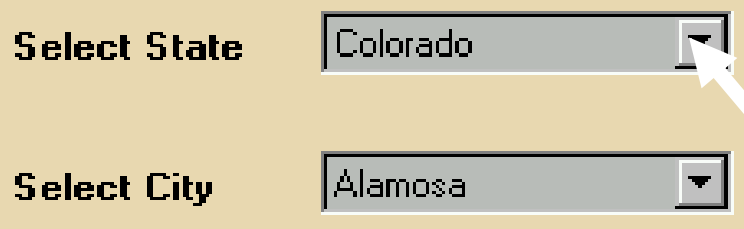

Select Month July

Select Day

Select Time $\quad 13$

Save Spectra to File Additional Information Return to VSOLE

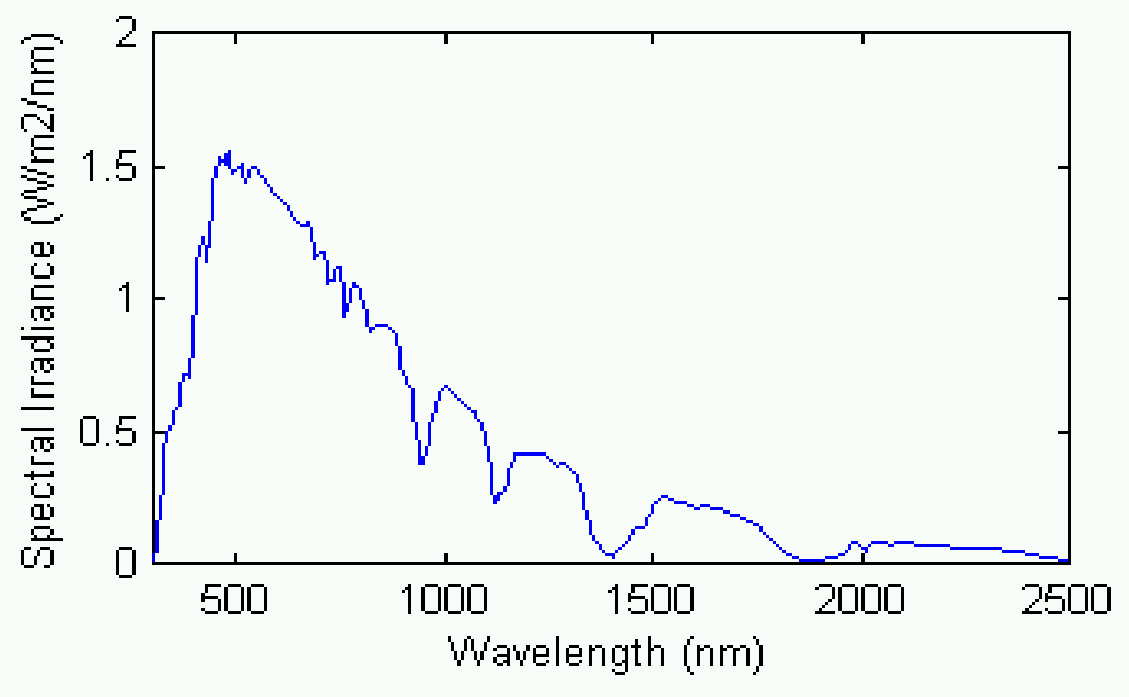

\section{Daily Value over a Month}

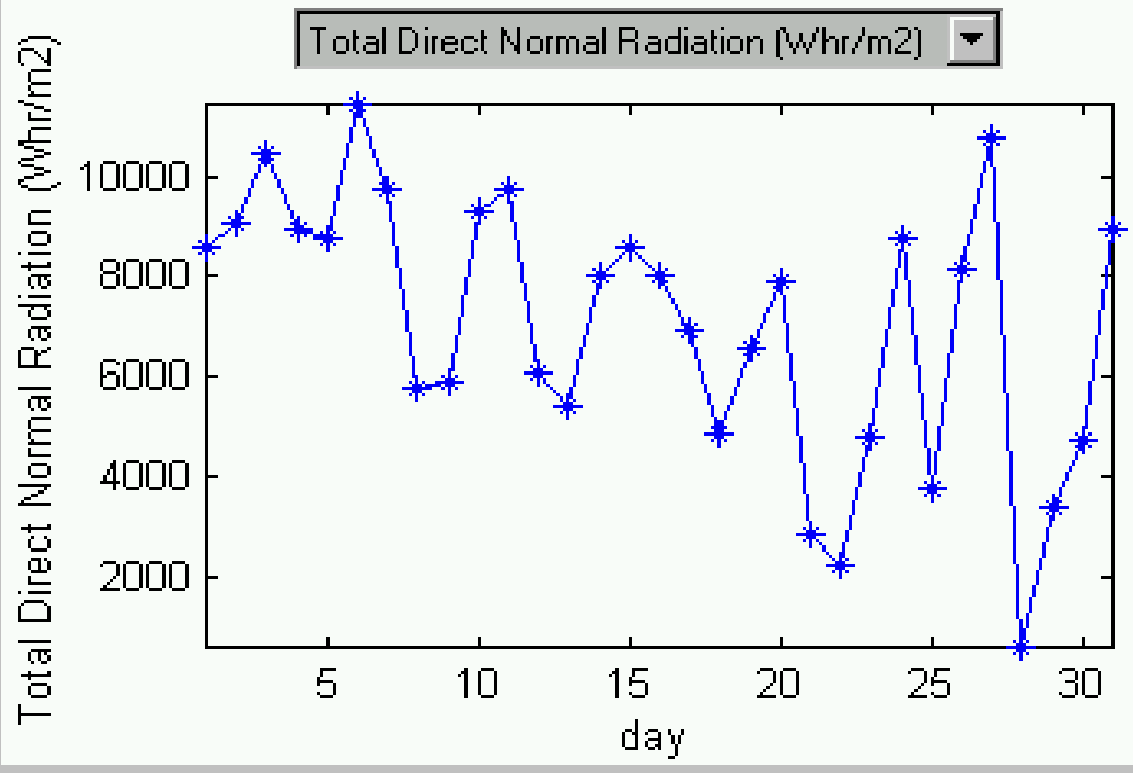

\section{Hourly Value over a Day}

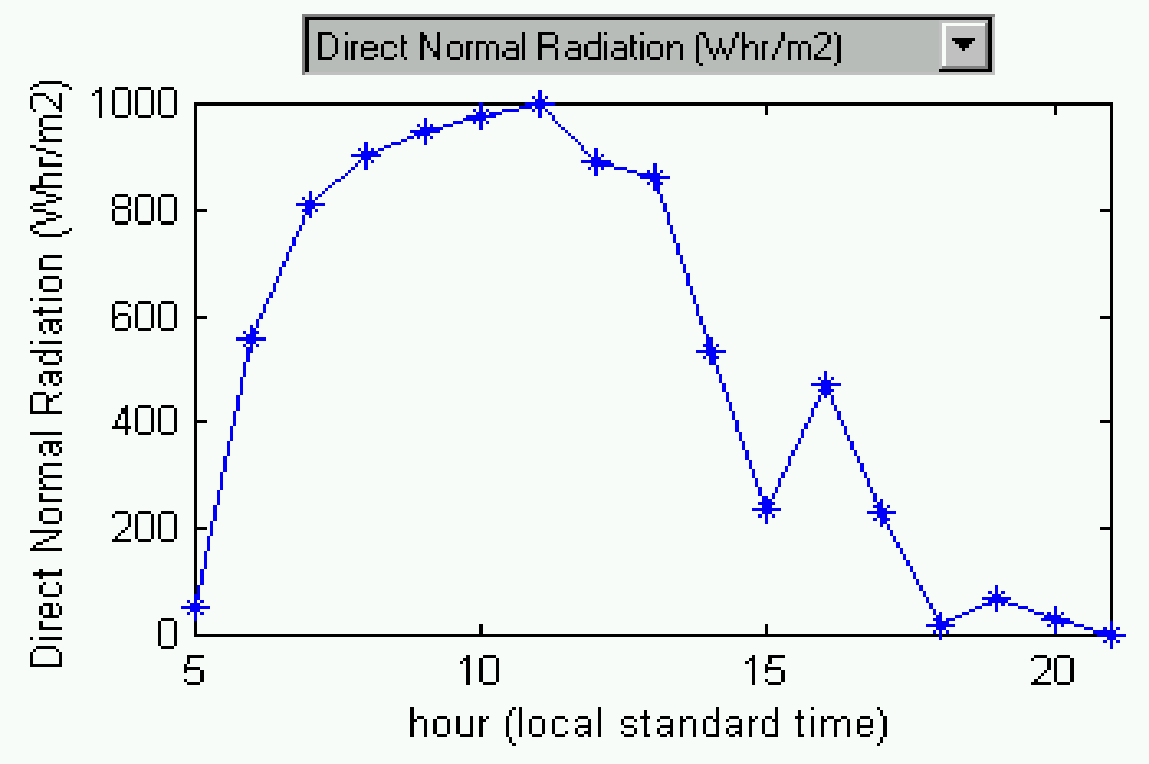




\section{Daily Value over a Month}

Colorado

Select State

Connecticut

Delaware

\section{Florida}

Georgia

Select City

Hawail

Illinois

Indiana

Select Month

lowa

Kansas

Kentucky

Louisiana

Maine

Maryland

Massachusetts

Michigan

Minnesota

Select Time

Mississippi

Missouri

Montana

Nebraska

Save Spectra to File

Nevada

A. New Hampshire

urn to VSOLE

New Mexic

New York

North Carolina

North Dakota

Ohio

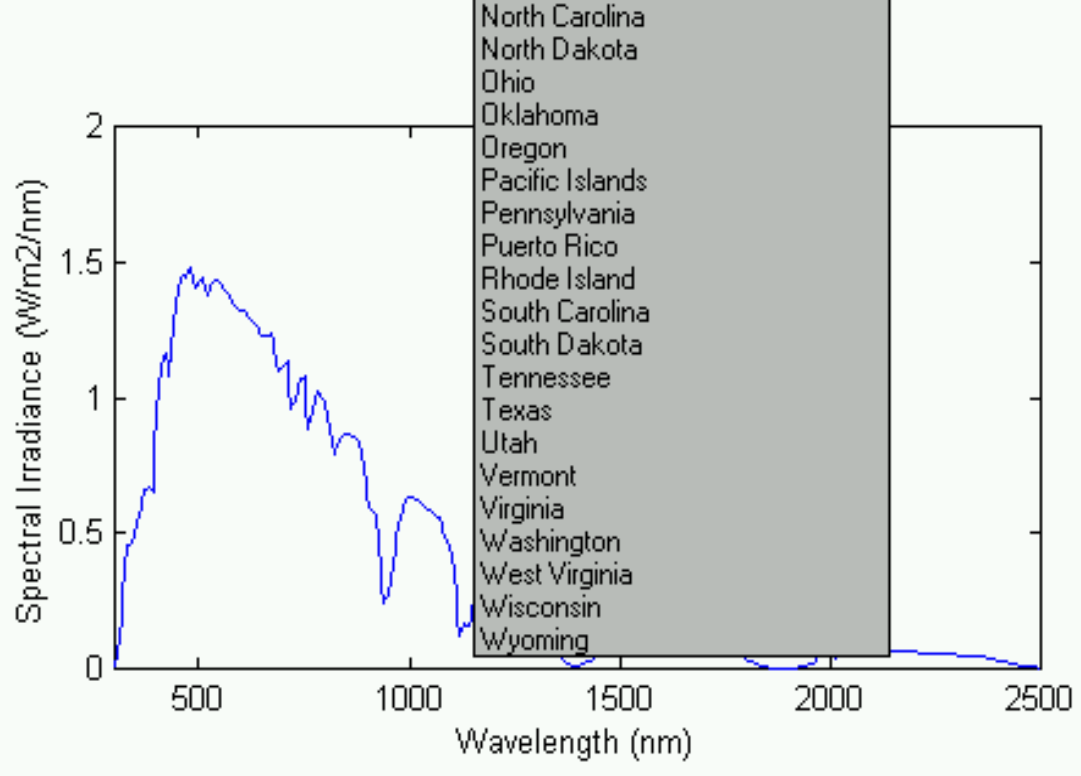

Total Direct Normal Radiation (W/hr/m2)

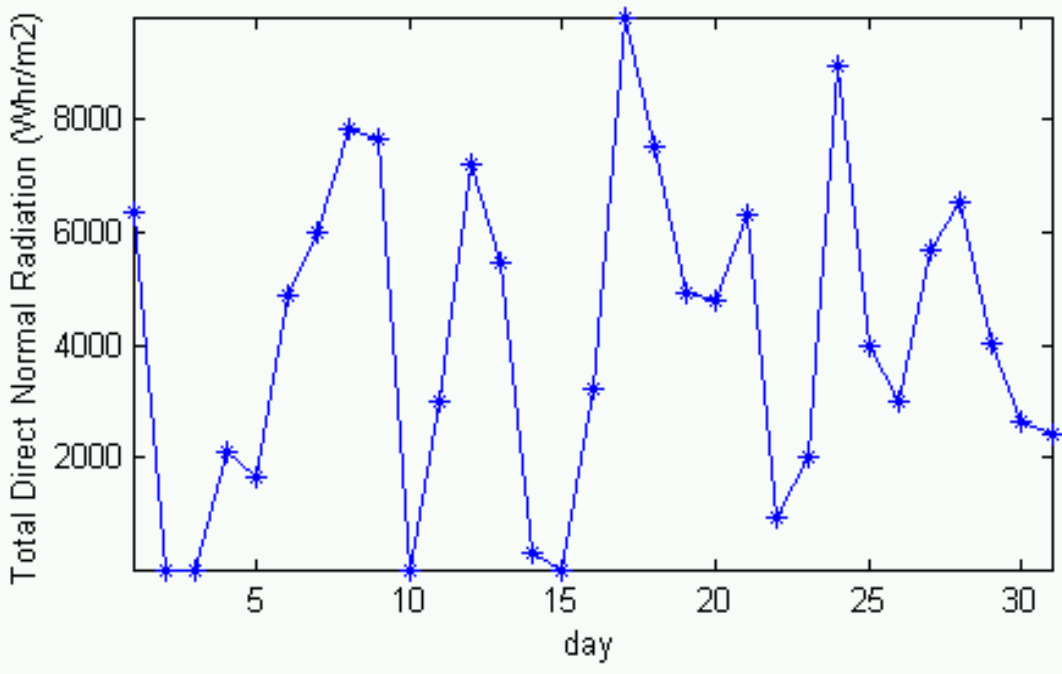

Hourly Value over a Day

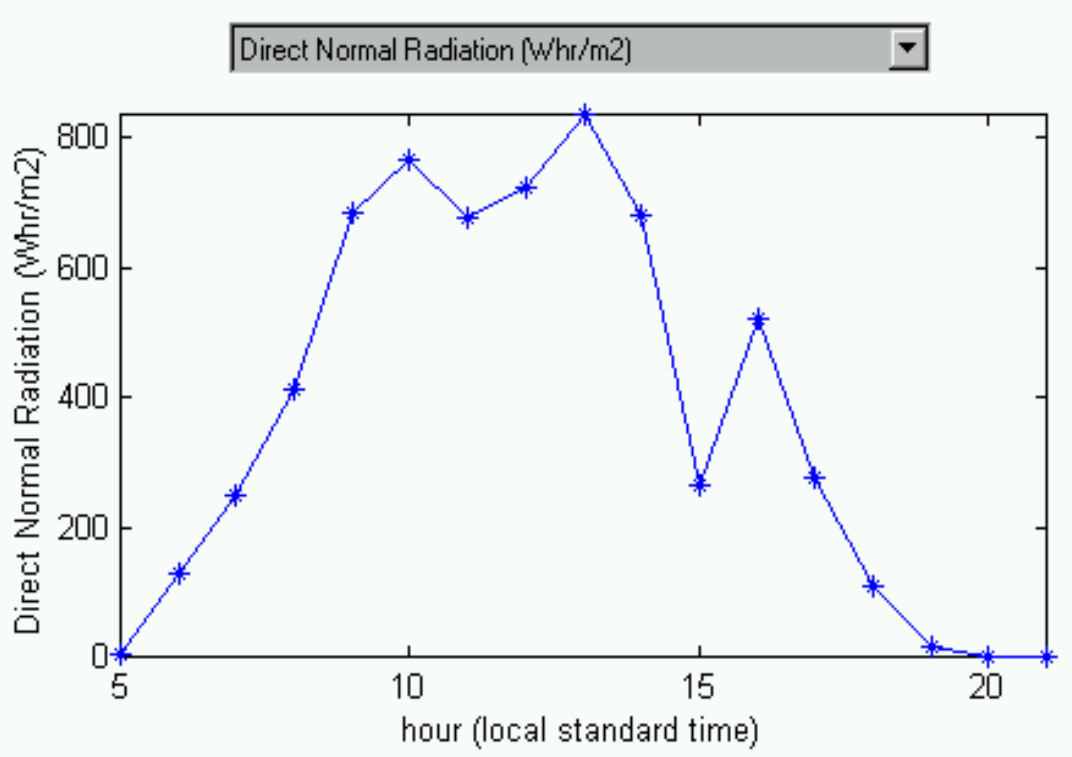




\begin{tabular}{|c|c|c|}
\hline Select State & Florida & $\nabla$ \\
\hline \multirow[t]{2}{*}{ Select City } & Miami & -7 \\
\hline & $\begin{array}{l}\text { Daytona Beach } \\
\text { Jacksonville } \\
\text { Key West }\end{array}$ & \\
\hline Select Month & $\begin{array}{l}\text { Deg west } \\
\text { Milami m. } \\
\text { Tallahassee m.m. }\end{array}$ & \\
\hline Select Day & West Palm Beach & \\
\hline
\end{tabular}

Select Time 13

Save Spectra to File Additional Information Return to VSOLE

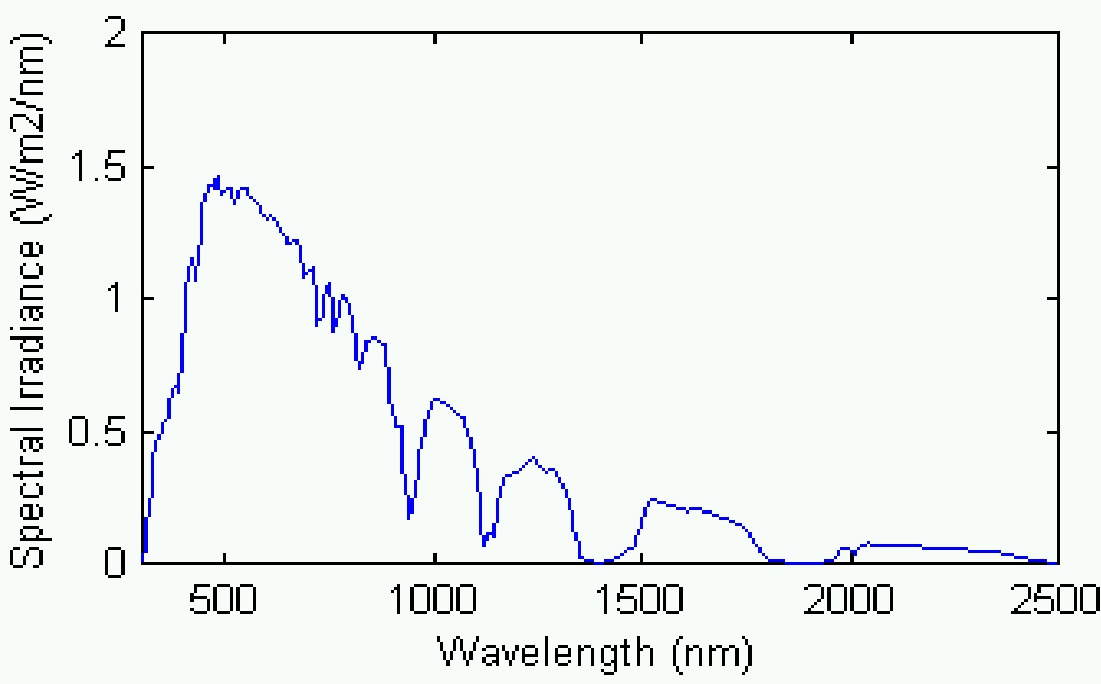

Daily Value over a Honth

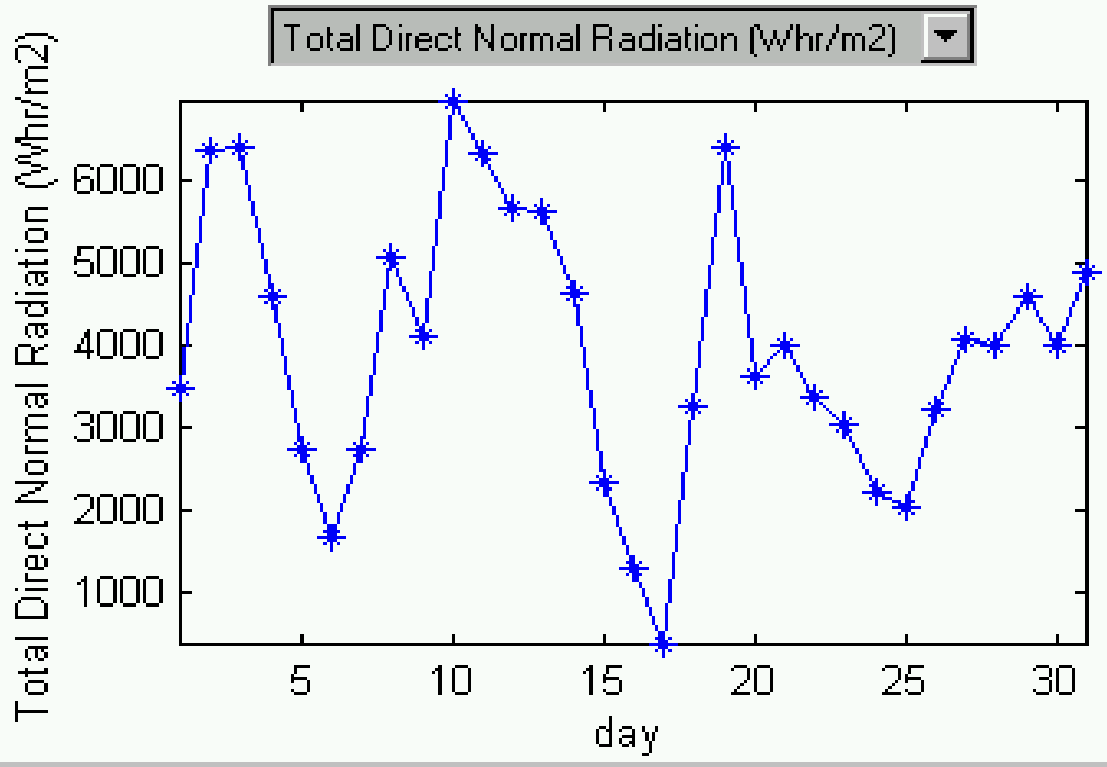

Hourly Value over a Day

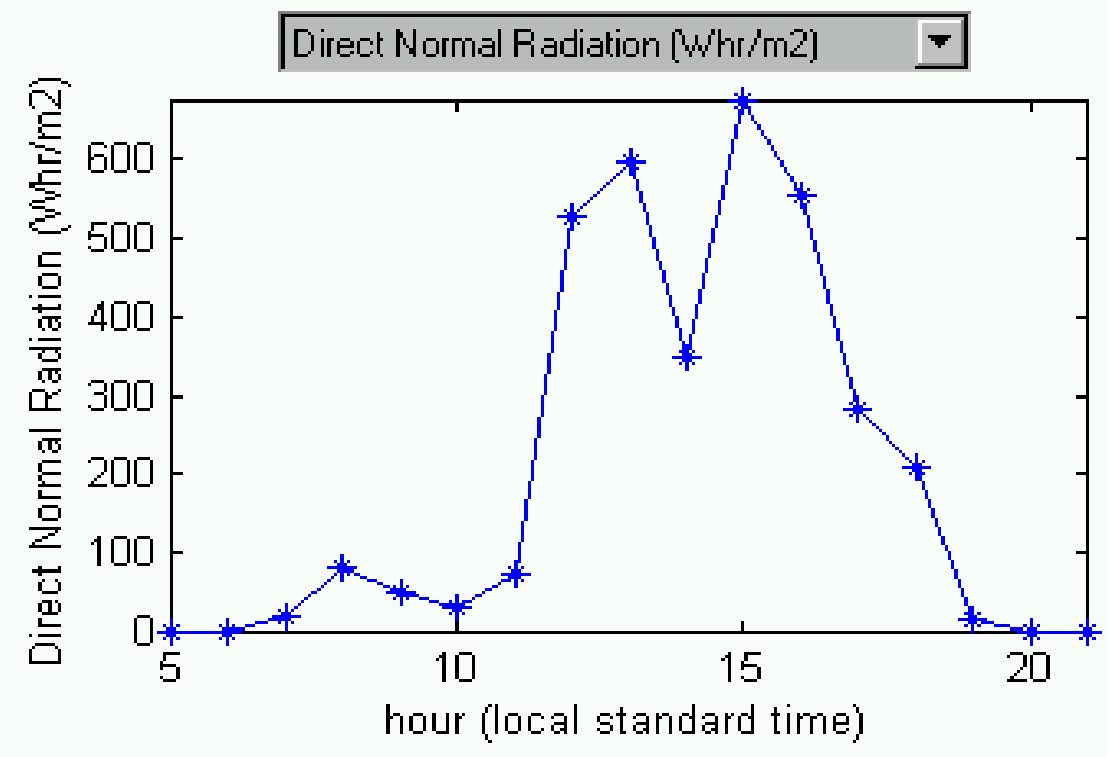



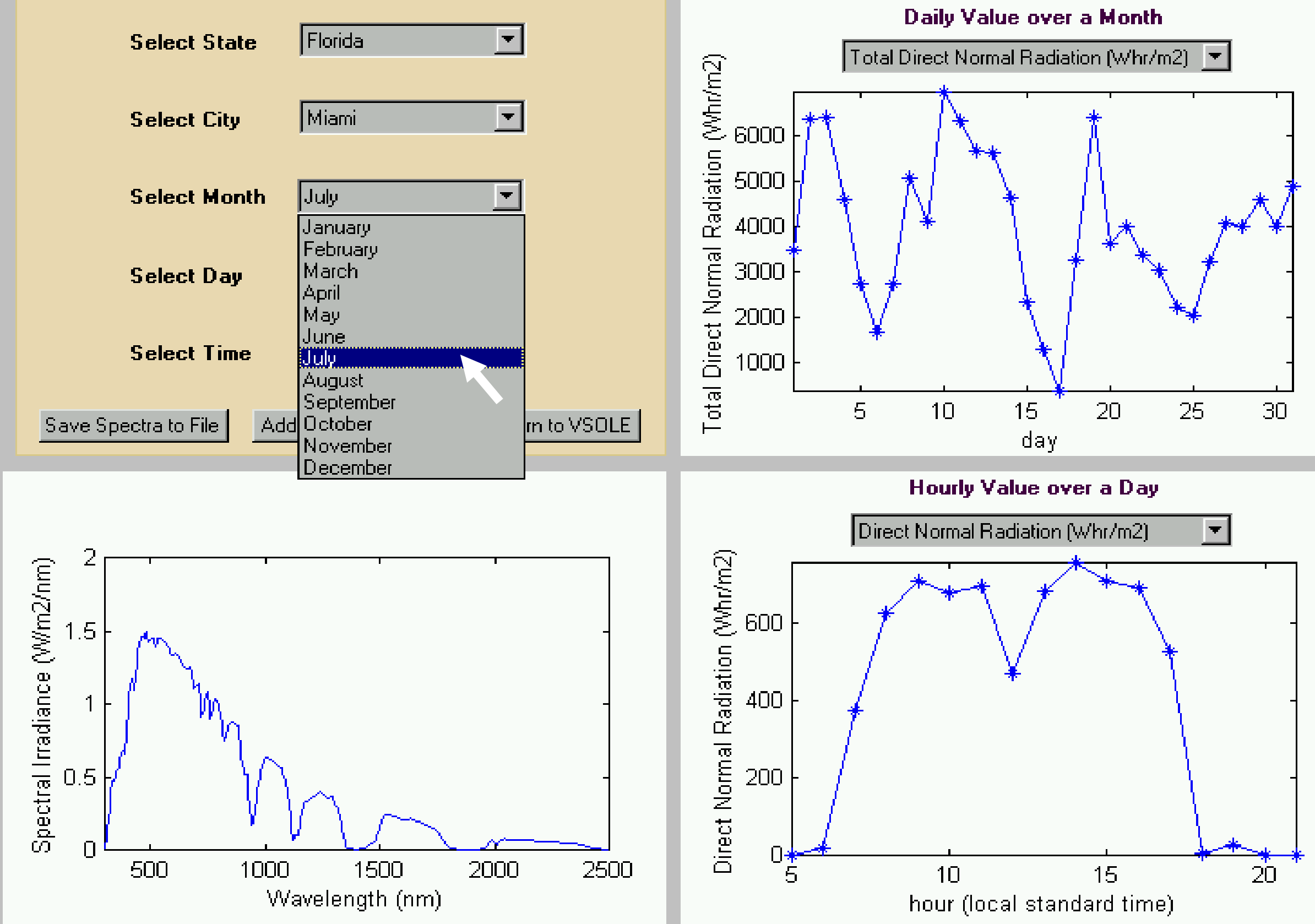


Select State Florida

Select City Miami

Select Month July

Select Day

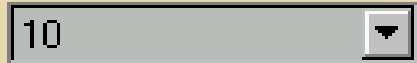

Select Time

$$
13
$$

Save Spectra to File Additional Information

Return to VSOLE

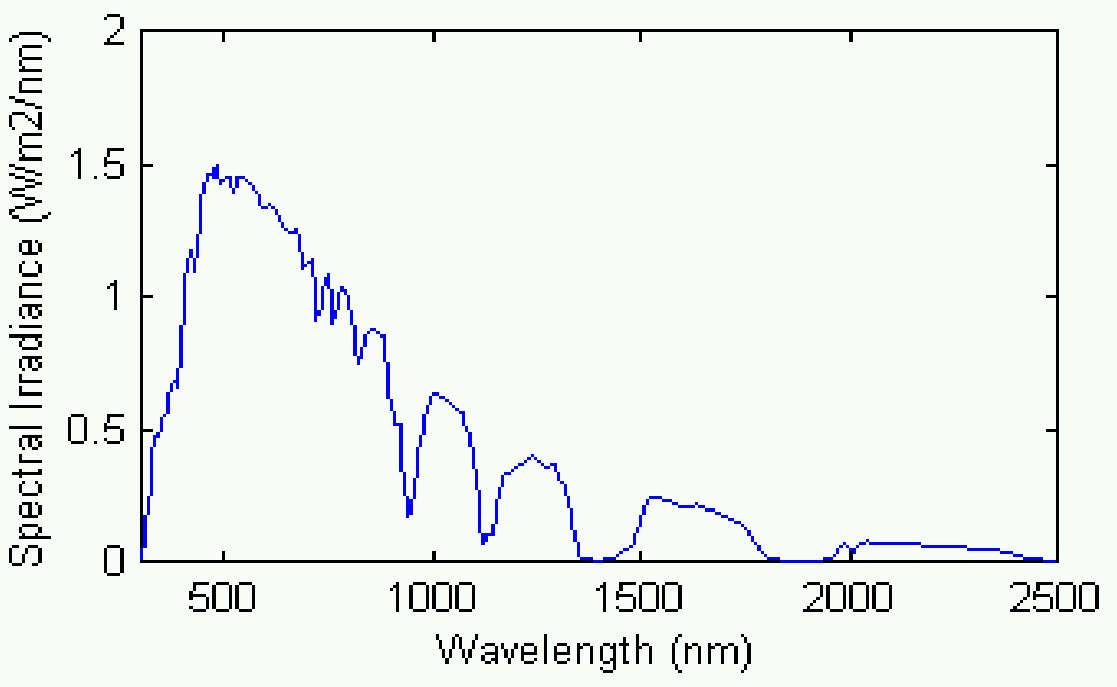

\section{Daily Value over a Month}

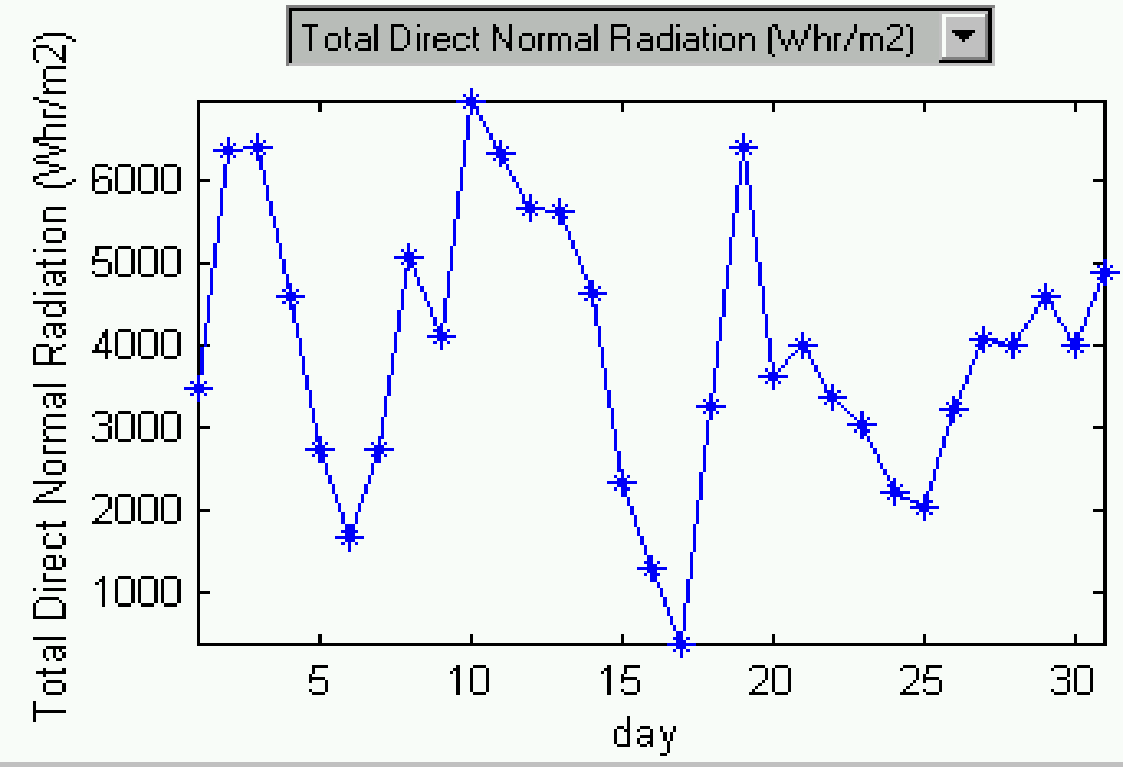

Hourly Value over a Day

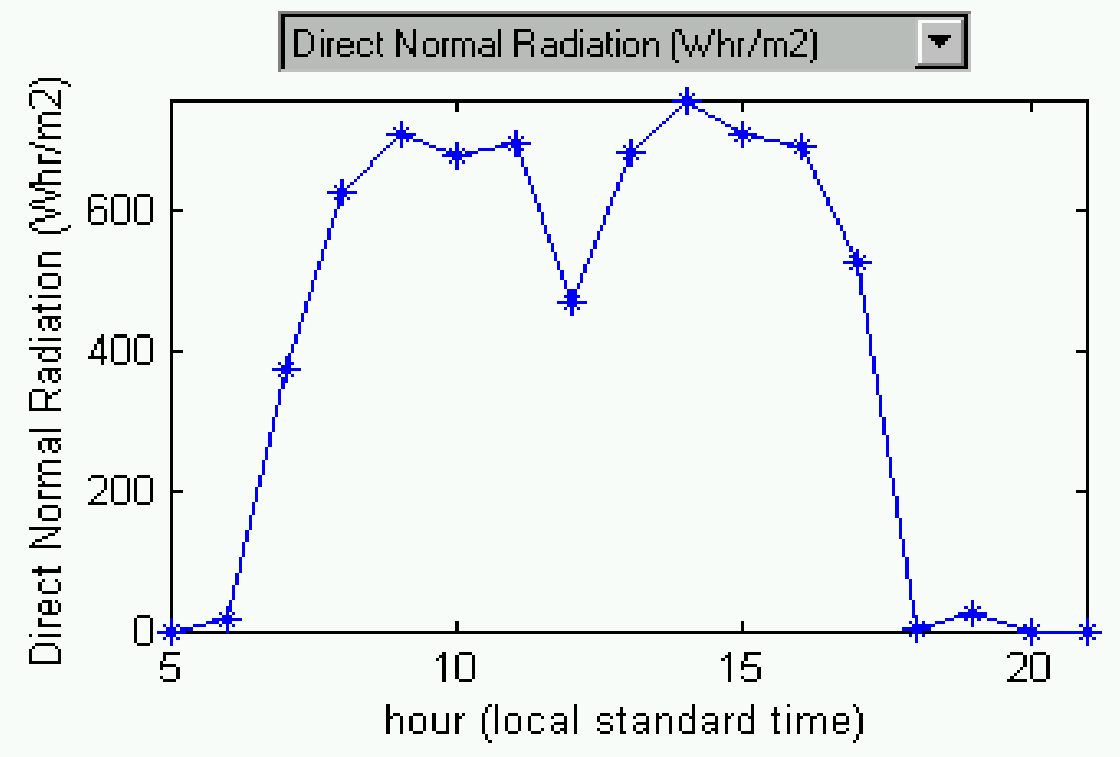




\section{Integrated Modeling}

Determine the solar load into your vehicle

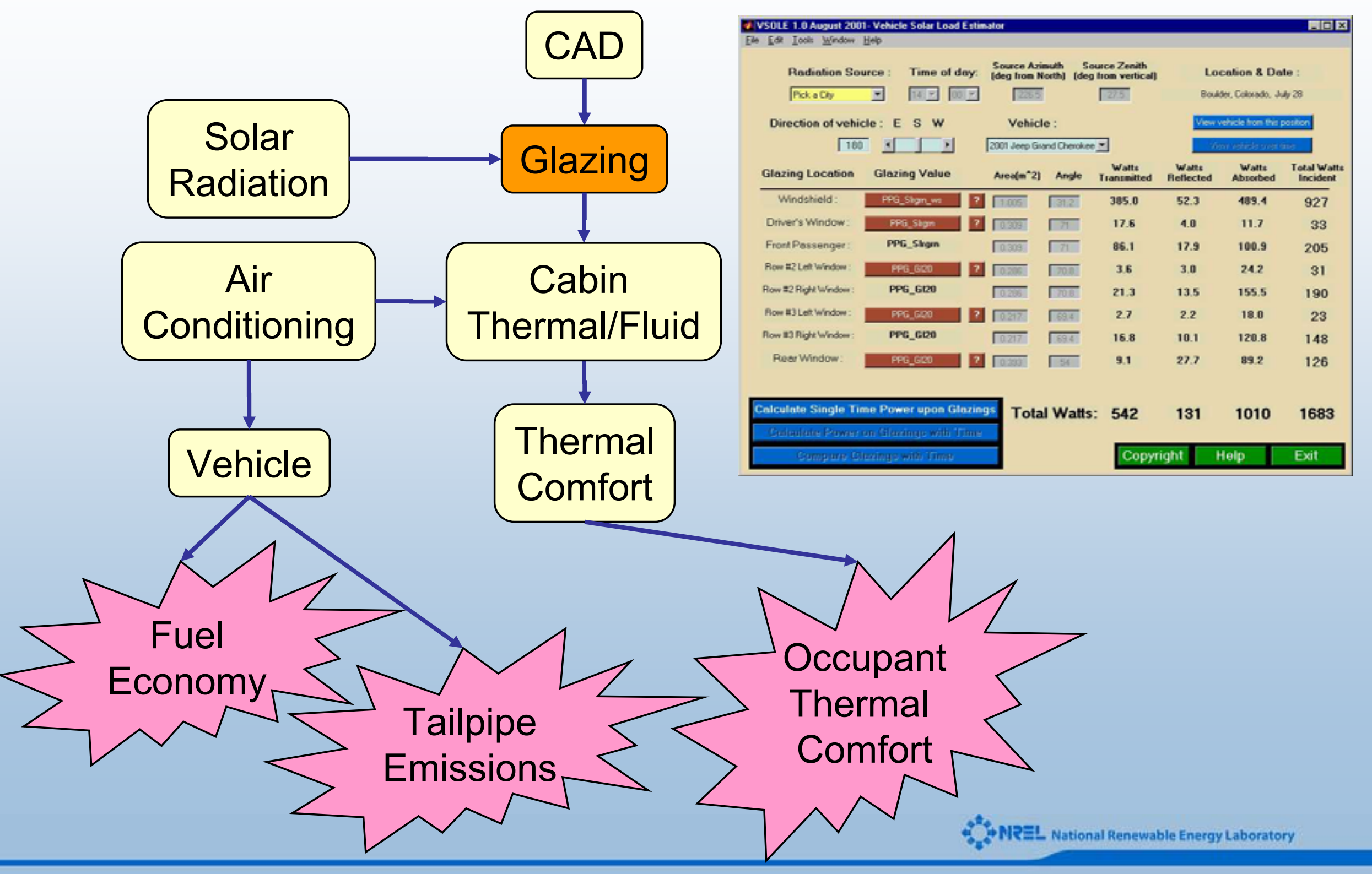


Radiation Source : Time of day:

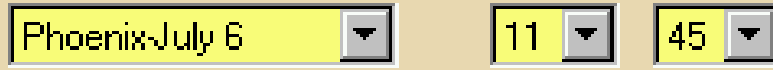

Source Azimuth [deg from North]
Source Zenith [deg from vertical]

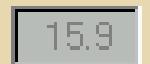

\section{Location \& Date :}

none selected
Direction of vehicle: $E S W$

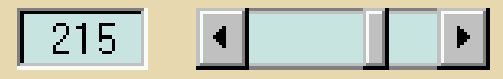

Vehicle :

Morph Vehicle $\quad$ 工

View vehicle from this position

View vehicle over time

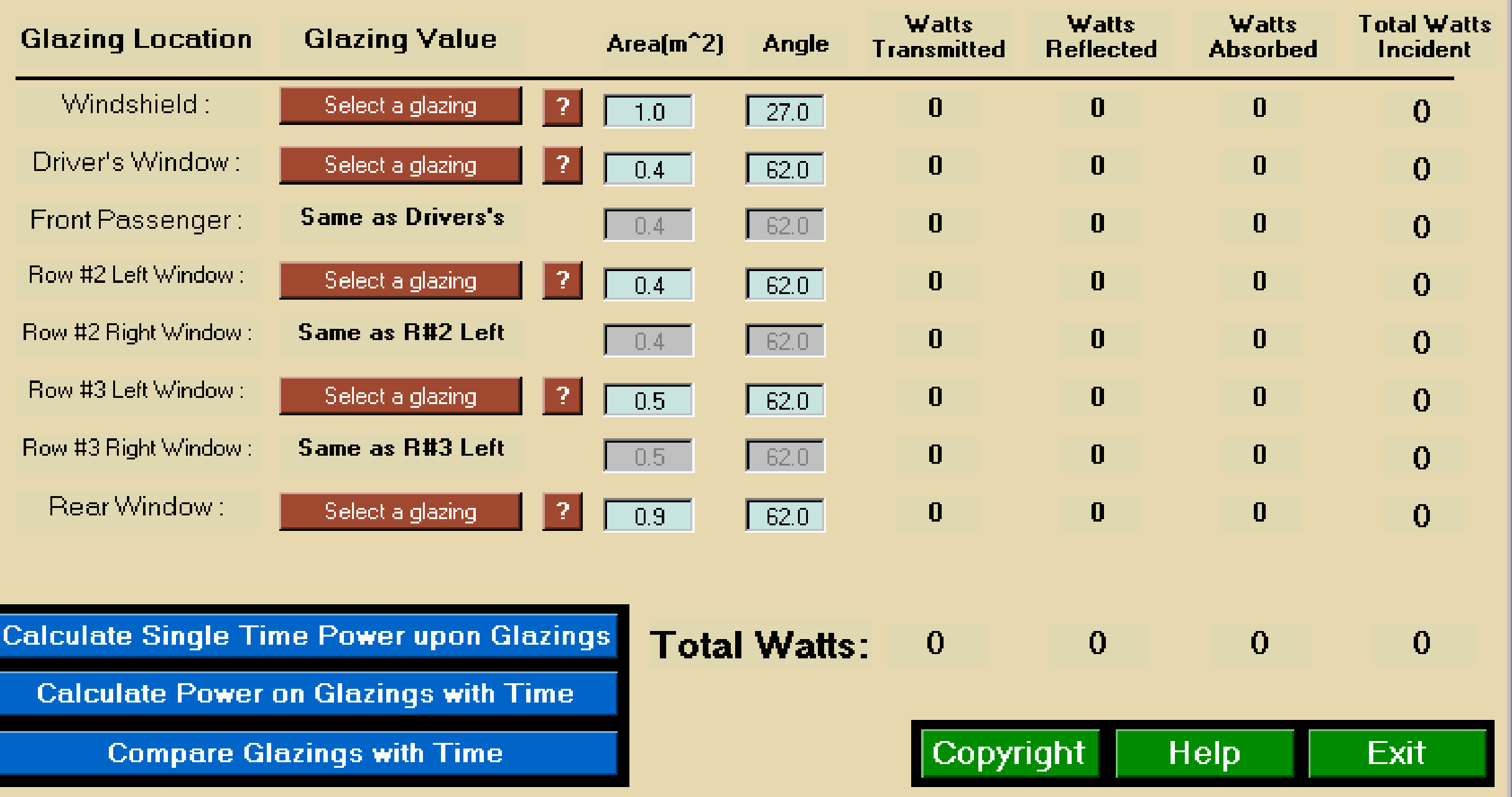


Radiation Source : Time of day:

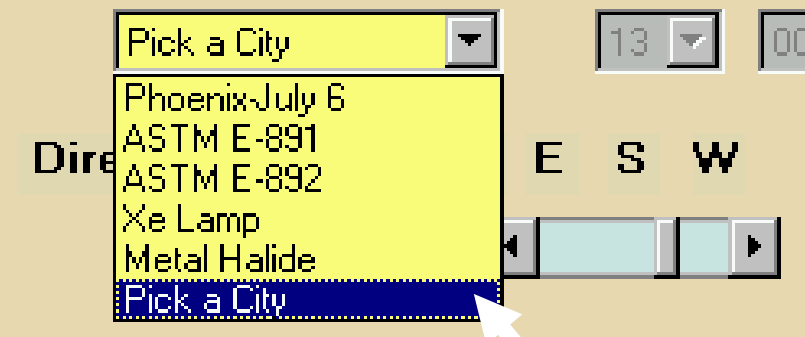

Glazing Location Glo ring Value
Source Azimuth [deg from North]

Source Zenith [deg from vertical]

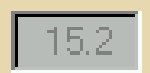

\section{Location \& Date:}

Alamosa, Colorado, July 1

View vehicle from this position

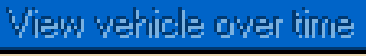

Morph Vehicle

watts watts wats Total watts Area[m^2] Angle Transmitted

\begin{tabular}{|c|c|c|c|c|c|c|c|}
\hline Glazing Location & Gla >ing Value & Area[m^2 & Angle & $\begin{array}{l}\text { Watts } \\
\text { Transmitted }\end{array}$ & $\begin{array}{l}\text { Watts } \\
\text { Reflected }\end{array}$ & $\begin{array}{l}\text { Watts } \\
\text { Absorbed }\end{array}$ & $\begin{array}{l}\text { Total Watt } \\
\text { Incident }\end{array}$ \\
\hline Windshield & Select a glazing & 1.0 & 27.0 & $\mathbf{0}$ & $\mathbf{0}$ & $\mathbf{0}$ & 0 \\
\hline Driver's Window: & Select a glazing & 0.4 & 62.0 & $\mathbf{0}$ & $\mathbf{0}$ & $\mathbf{0}$ & 0 \\
\hline Front Passenger: & Same as Drivers's & 0.4 & 62.0 & $\mathbf{0}$ & $\mathbf{0}$ & $\mathbf{0}$ & 0 \\
\hline Row $\# 2$ Left Window: & Select a glazing & 0.4 & 62.0 & $\mathbf{0}$ & $\mathbf{0}$ & $\mathbf{0}$ & 0 \\
\hline Row \#2 Right Window: & Same as R\#2 Left & 0.4 & 62.0 & $\mathbf{0}$ & $\mathbf{0}$ & 0 & 0 \\
\hline Row \#3 Left Window: & Select a glazing & 0.5 & 62.0 & $\mathbf{0}$ & $\mathbf{0}$ & $\mathbf{0}$ & 0 \\
\hline Row \#3 Right Window: & Same as R\#3 Left & 0.5 & 62.0 & $\mathbf{0}$ & $\mathbf{0}$ & $\mathbf{0}$ & 0 \\
\hline Rear Window: & Select a glazing & 0.9 & 62.0 & 0 & 0 & 0 & 0 \\
\hline
\end{tabular}


Radiation Source: Time of day:

Pick a City

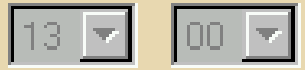

Source Azimuth [deg from North]

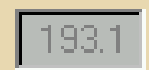

Source Zenith [deg from vertical]

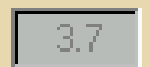

\section{Location \& Date:}

Miami, Florida, July 10
Direction of vehicle: $E S W$

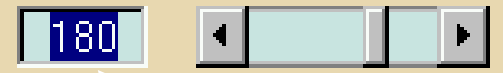

Vehicle :

Morph Vehicle $\quad$ -

View vehicle from this position

Yiem vehiche over tifne Area[m^2] Angle $\begin{gathered}\text { Watts } \\ \text { Transmitted }\end{gathered} \quad \begin{gathered}\text { Watts } \\ \text { Reflected }\end{gathered} \begin{gathered}\text { Watts } \\ \text { Absorbed }\end{gathered} \quad \begin{gathered}\text { Total Watts } \\ \text { Incident }\end{gathered}$

\begin{tabular}{|c|c|c|c|c|c|c|c|c|}
\hline Glazing Location & Glazing Value & & Area $\left[m^{\wedge} 2\right]$ & Angle & $\begin{array}{c}\text { Watts } \\
\text { Transmitted }\end{array}$ & $\begin{array}{c}\text { Watts } \\
\text { Reflected }\end{array}$ & $\begin{array}{c}\text { Watts } \\
\text { Absorbed }\end{array}$ & $\begin{array}{l}\text { Total watt } \\
\text { Incident }\end{array}$ \\
\hline Windshield: & Select a glazing & $?$ & 1.0 & 27.0 & $\mathbf{0}$ & 0 & 0 & 0 \\
\hline Driver's Window: & Select a glazing & $?$ & 0.4 & 62.0 & $\mathbf{0}$ & $\mathbf{0}$ & $\mathbf{0}$ & 0 \\
\hline Front Passenger: & Same as Drivers's & & 0.4 & 62.0 & $\mathbf{0}$ & $\mathbf{0}$ & $\mathbf{0}$ & 0 \\
\hline Row \#2 Left Window : & Select a glazing & $?$ & 0.4 & 62.0 & $\mathbf{0}$ & $\mathbf{0}$ & $\mathbf{0}$ & 0 \\
\hline Row \#2 Right Window: & Same as R\#2 Left & & 0.4 & 62.0 & $\mathbf{0}$ & $\mathbf{0}$ & $\mathbf{0}$ & 0 \\
\hline Row \#3 Left Window: & Select a glazing & $?$ & 0.5 & 62.0 & 0 & $\mathbf{0}$ & 0 & 0 \\
\hline Row \#3 Right Window : & Same as R\#3 Left & & 0.5 & 62.0 & 0 & $\mathbf{0}$ & 0 & 0 \\
\hline Rear Window: & Select a glazing & $?$ & 0.9 & 62.0 & 0 & $\mathbf{0}$ & 0 & 0 \\
\hline
\end{tabular}


Radiation Source : Time of day:

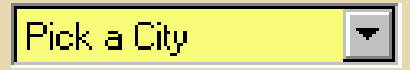

Source Azimuth [deg from North]

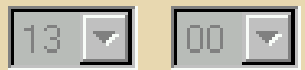

Source Zenith [deg from vertical]

\section{Location \& Date:}

Miami, Florida, July 10
Direction of vehicle: $E S W$

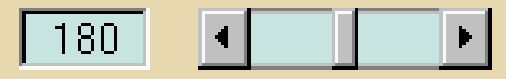

\section{Glazing Location Glazing Value}

Windshield:

Select a glazing

? 2001 Jeep Grand Cherokee

Driver's Window:

Front Passenger:

Row \#2 Left Window:

Row \#2 Right Window:

Row \#3 Left Window:

Row \#3 Right Window:

Rear Window:

\begin{tabular}{c|} 
Select a glazing \\
\hline Same as Drivers's \\
Select a glazing \\
\hline
\end{tabular}

Same as R\#2 Left

Select a glazing

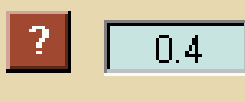

Morph Vehicle Morph Vehicle 2001 Honda Insight 2001 Toyota Prius 1998 Chrysler Minivan

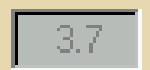

Vehicle :

View vehicle from this position

Viem vehricie over tinie

watts Watts wats Total watts ansmitted Reflected Absorbed Incident

Same as R\#3 Left

Select a glazing

? 0.9

62.0

$\longdiv { 6 2 . 0 }$

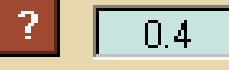

62.0

0

0

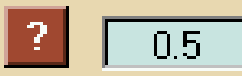

$\longdiv { 6 2 . 0 }$

0

0

0

Total Watts:

0

0

0 
Radiation Source : Time of day:

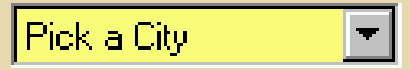

Source Azimuth [deg from North]

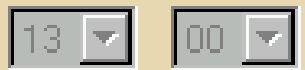

Source Zenith [deg from vertical]

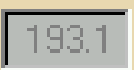

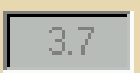

\section{Location \& Date:}

Miami, Florida, July 10
Direction of vehicle: $E S W$

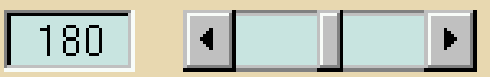

Vehicle :

2001 Jeep Grand Cherokee $\nabla$
View vehicle from this position

Vien vericite ayer tinte

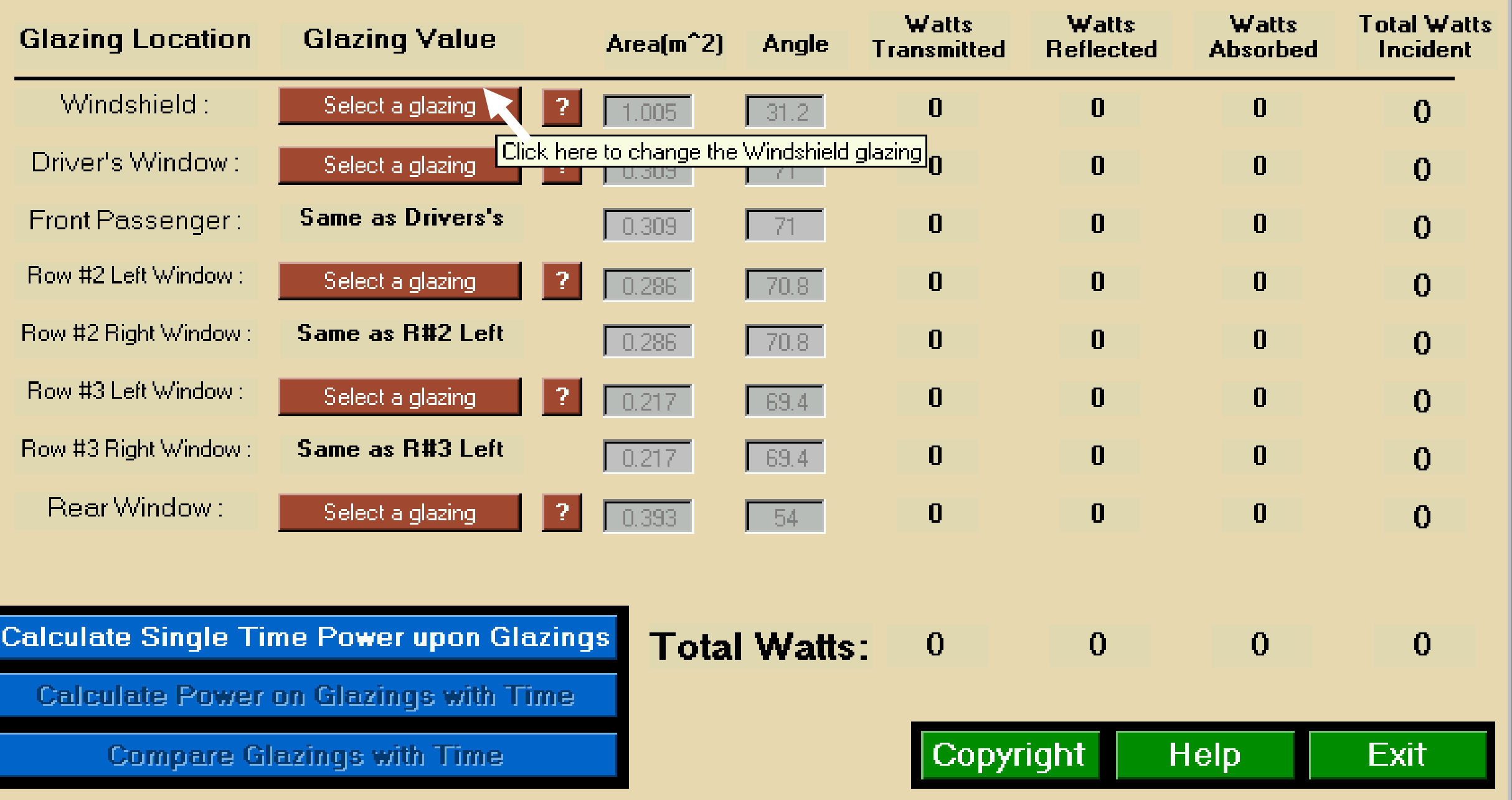


Radiation Source : Time of day:

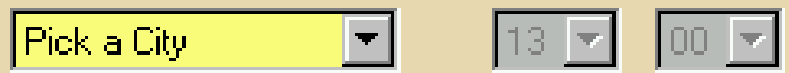

Source Azimuth [deg from North]
Source Zenith [deg from vertical]

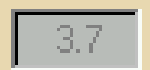

\section{Location \& Date:}

Miami, Florida, July 10

\section{Direction of vehicle : $E S W$ \\ 180 \\ Choose a glazing data file Look in: $\quad$ PPG_Glazings \\ Vehicle :}

\section{Glazing Location}

Windshield:

Driver's Window:

Front Passenger :

Row \#2 Left Window:

Row \#2 Right Window:

Row \#3 Left Window:

Row \#3 Right Window:

Rear Window: Look in: 9 PP VPPG_Sgte_eu_ws.dat 20)PPG_Sgte_us_ws.dat

- 0 PPG_SIrgrn_ws.dat 김PG_Solex_ws.dat - 0 PPG_Sxtra_ws.dat

\section{Same}

Seled

File name:

Files of type: ${ }^{\text {_-ws.dat }}$

Same :

wehicle from this position

Viem vehricie over tinie

Watts
eflected $\begin{gathered}\text { Watts } \\ \text { Absorbed }\end{gathered} \quad \begin{gathered}\text { Total Watts } \\ \text { Incident }\end{gathered}$

000

0

0

0

0

0

0

0

0

0

0

\begin{abstract}
Select a glazing
\end{abstract}
Same as R\#3 Left

Select a glazing

\section{?] $\longdiv { 0 . 2 1 7 }$}

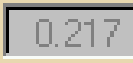

$? \longdiv { 0 . 3 9 3 }$

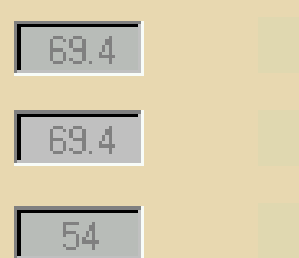

0

0

0
Total Watts: 0
0

0 
Radiation Source: Time of day:

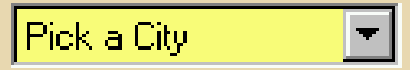

Source Azimuth [deg from North]

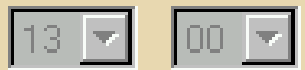

Source Zenith [deg from vertical]

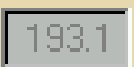

\section{Location \& Date:}

Miami, Florida, July 10
Direction of vehicle: $E S W$

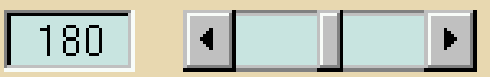

Vehicle :

2001 Jeep Grand Cherokee $\nabla$
View vehicle from this position

View vehicite over tinne Area $\left[\mathrm{m}^{\wedge} 2\right]$ Angle $\begin{gathered}\text { Watts } \\ \text { Transmitted }\end{gathered} \quad \begin{gathered}\text { Watts } \\ \text { Reflected }\end{gathered} \begin{gathered}\text { Watts } \\ \text { Absorbed }\end{gathered} \quad \begin{gathered}\text { Total Watts } \\ \text { Incident }\end{gathered}$

\begin{tabular}{|c|c|c|c|c|c|c|c|c|}
\hline Glazing Location & Glazing Value & & Area $\left[\mathrm{m}^{\wedge} 2\right]$ & Angle & $\begin{array}{c}\text { Watts } \\
\text { Transmitted }\end{array}$ & $\begin{array}{c}\text { Watts } \\
\text { Reflected }\end{array}$ & $\begin{array}{c}\text { Watts } \\
\text { Absorbed }\end{array}$ & $\begin{array}{c}\text { Total Watt } \\
\text { Incident }\end{array}$ \\
\hline Windshield & PPG_Sgte_us_ws & $?$ & 1.005 & 31.2 & 0 & 0 & 0 & 0 \\
\hline Driver's Window: & PPG_Slrgrn & $?$ & 0.309 & 71 & $\mathbf{0}$ & $\mathbf{0}$ & $\mathbf{0}$ & 0 \\
\hline FrontPassenger: & PPG_Sirgrn & & 0.309 & 71 & $\mathbf{0}$ & $\mathbf{0}$ & $\mathbf{0}$ & 0 \\
\hline Row \#2 Left Window: & PPG_Gl20 & $?$ & $\begin{array}{l}0.286 \\
\end{array}$ & 70.8 & $\mathbf{0}$ & 0 & 0 & 0 \\
\hline Row \#2 Right Window: & PPG_GI20 & & $\lcm{0.286}$ & 70.8 & $\mathbf{0}$ & $\mathbf{0}$ & $\mathbf{0}$ & 0 \\
\hline Row \#3 Left Window: & PPG_Gl20 & $?$ & 0.217 & 69.4 & $\mathbf{0}$ & $\mathbf{0}$ & 0 & 0 \\
\hline Row \#3 Right Window : & PPG_GI20 & & 0.217 & 69.4 & $\mathbf{0}$ & 0 & $\mathbf{0}$ & 0 \\
\hline Rear Window: & PPG_GI20 & $?$ & 0.393 & 54 & $\mathbf{0}$ & 0 & 0 & 0 \\
\hline
\end{tabular}


Radiation Source: Time of day:

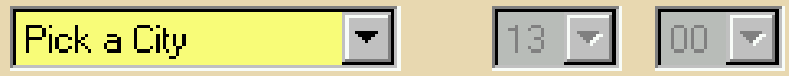

Source Azimuth Source Zenith [deg from North] [deg from vertical]

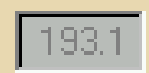

Location \& Date :

Miami, Florida, July 10
Direction of vehicle: $E S W$

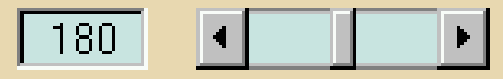

Vehicle:

2001 Jeep Grand Cherokee $\nabla$
View vehicle from this position

Vien vericie gyer tinte

Glazing Location Glazing Value Area[m^2] Angle $\begin{gathered}\text { watts } \\ \text { Transmitted }\end{gathered}$

watts Reflected

Watts Total watts Absorbed Incident

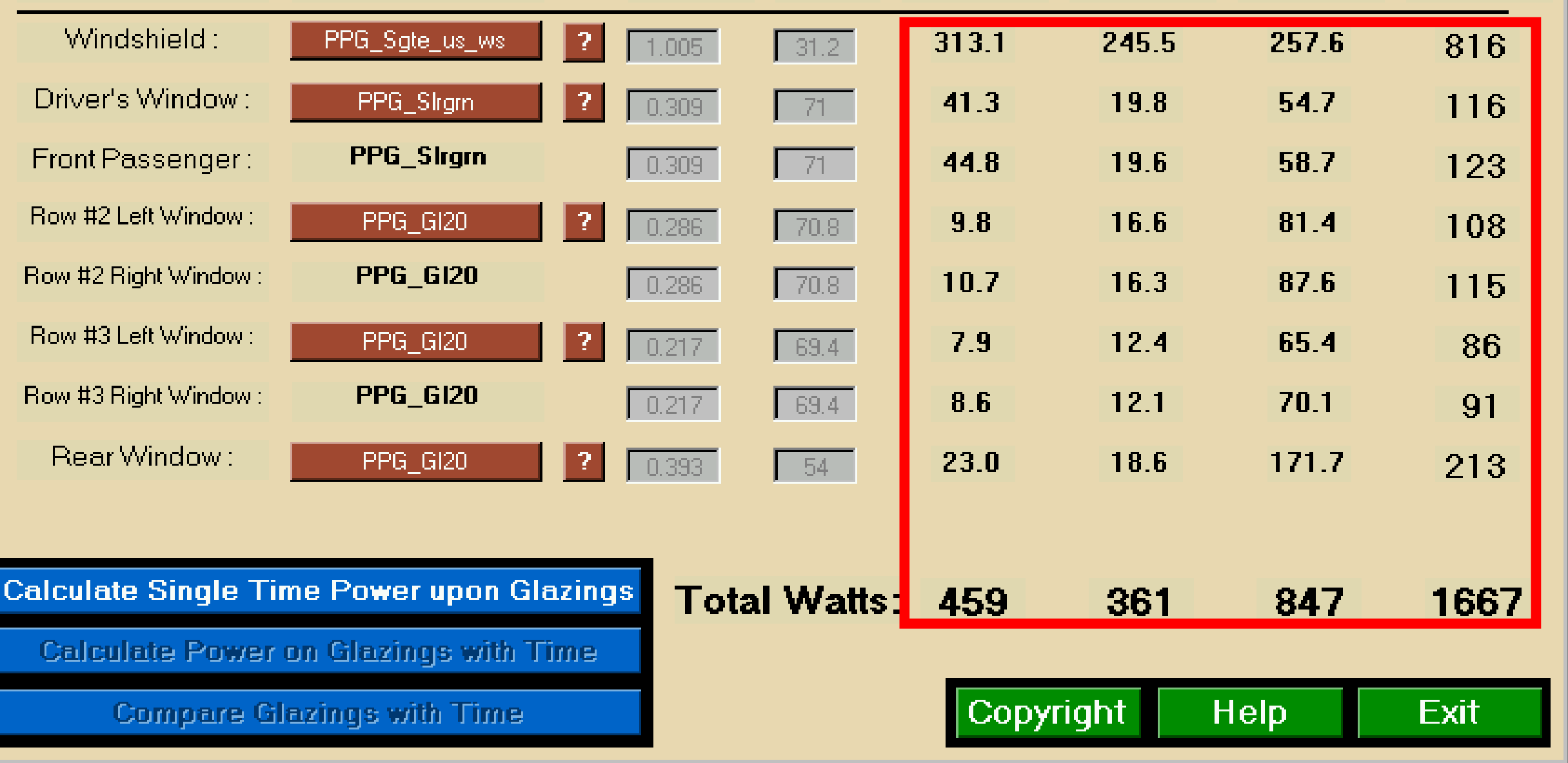




\section{Integrated Modeling}

Model temperatures and airflow in the cabin

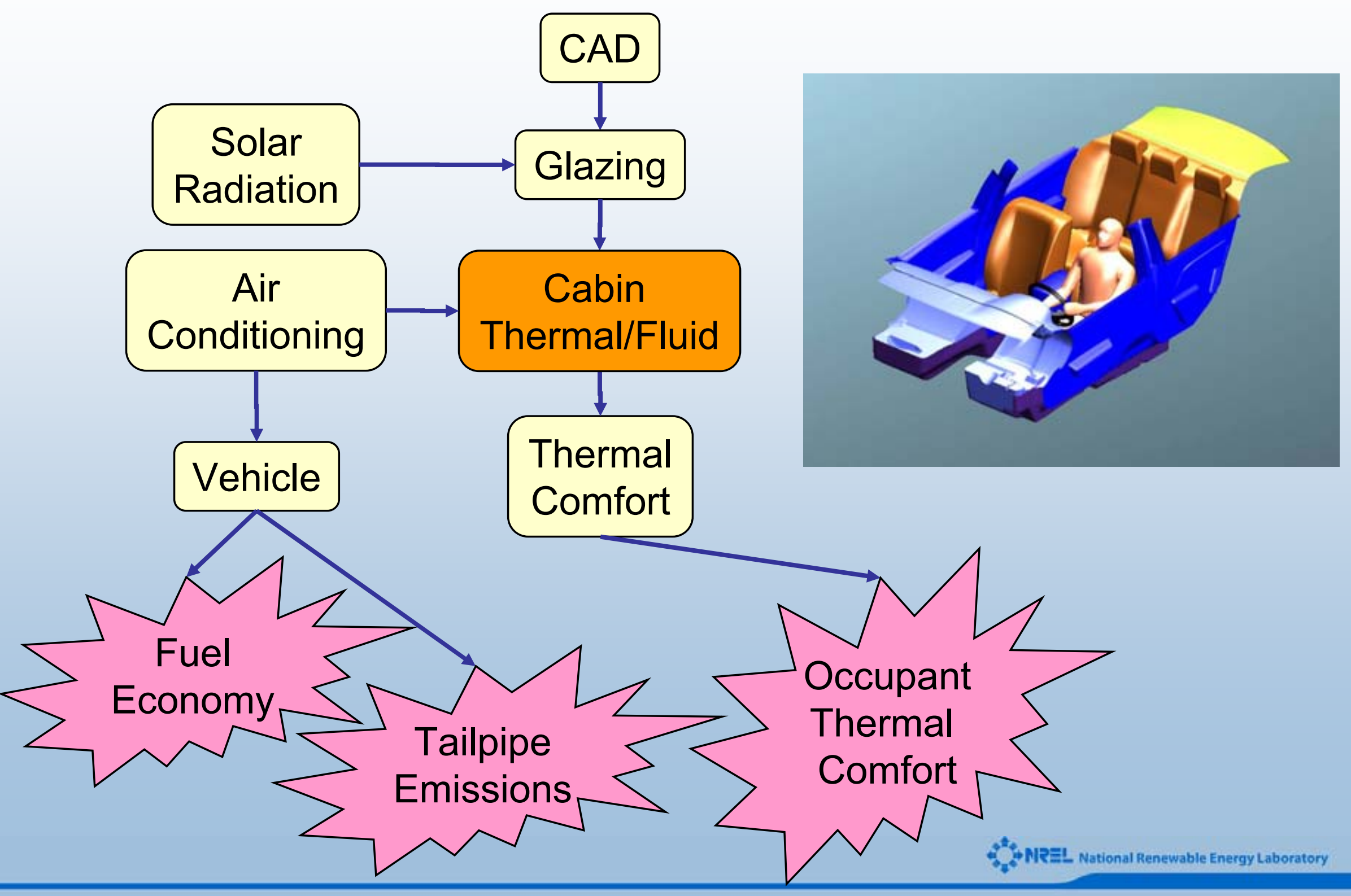




\section{Integrated Modeling}

Find the power consumption and cooling capacity of the $A / C$

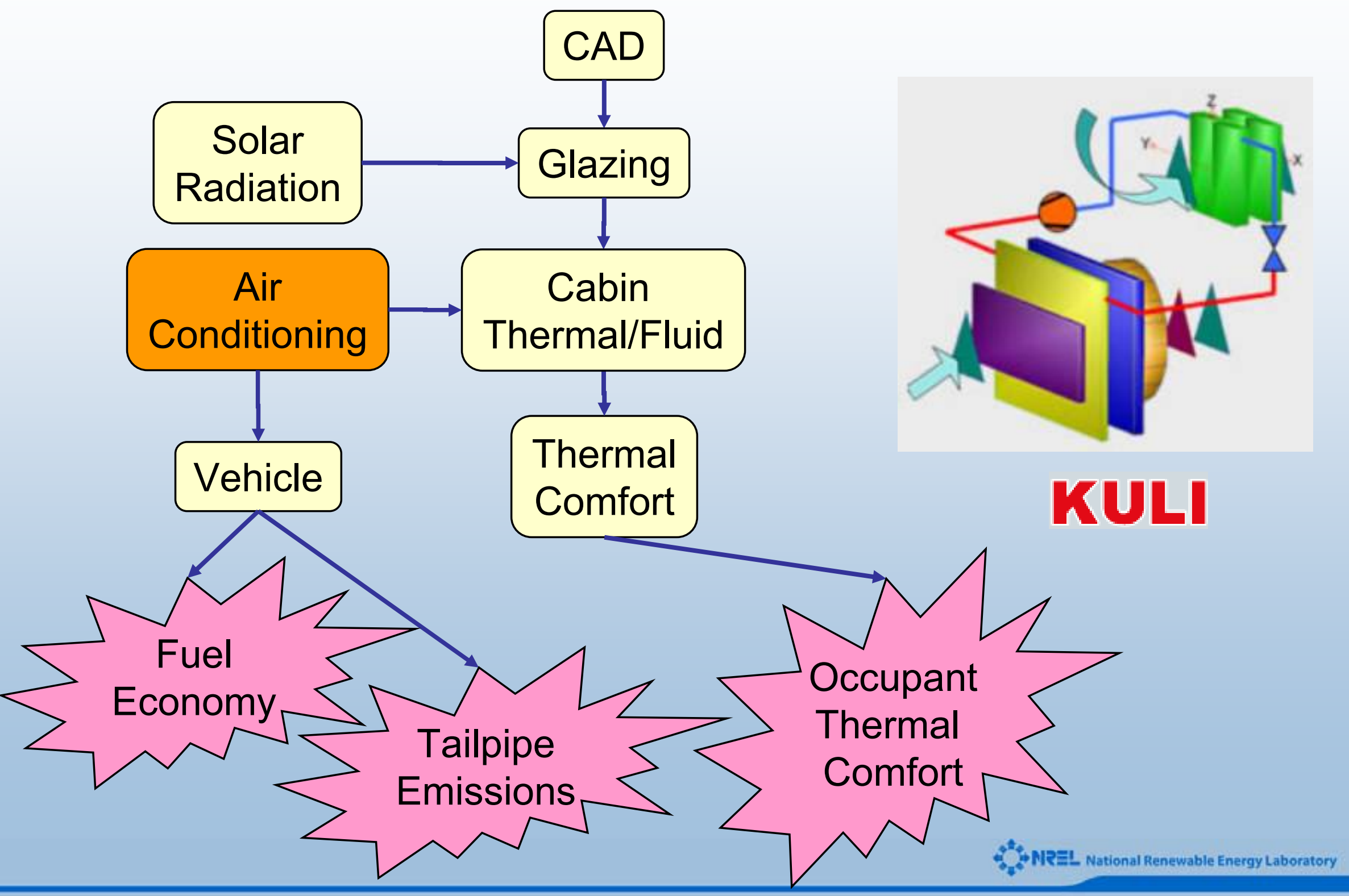




\section{Integrated Modeling}

How comfortable are you inside your car?

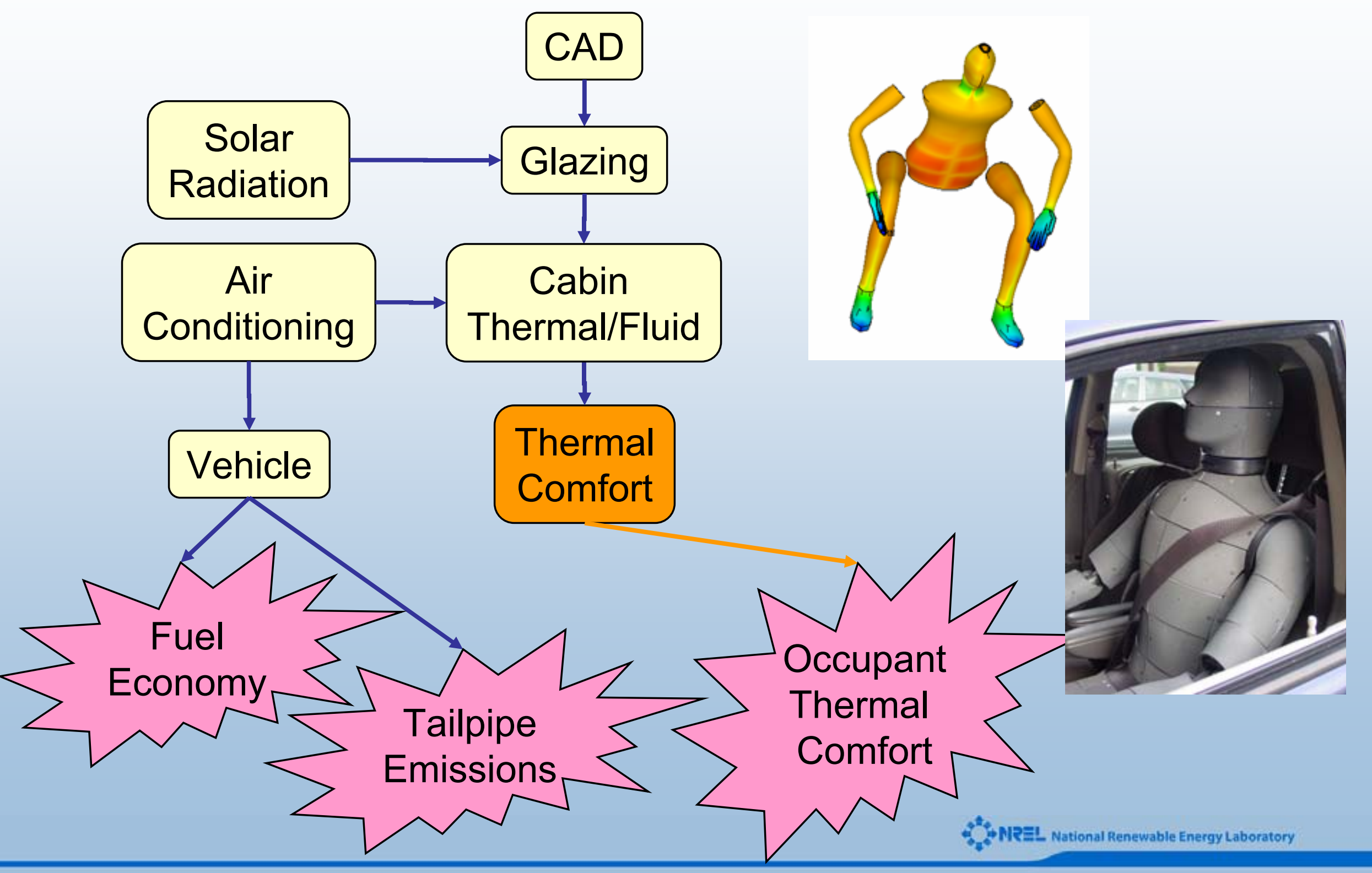


Thermal Comfort Assessment Tools Predicting the comfort of vehicle occupants

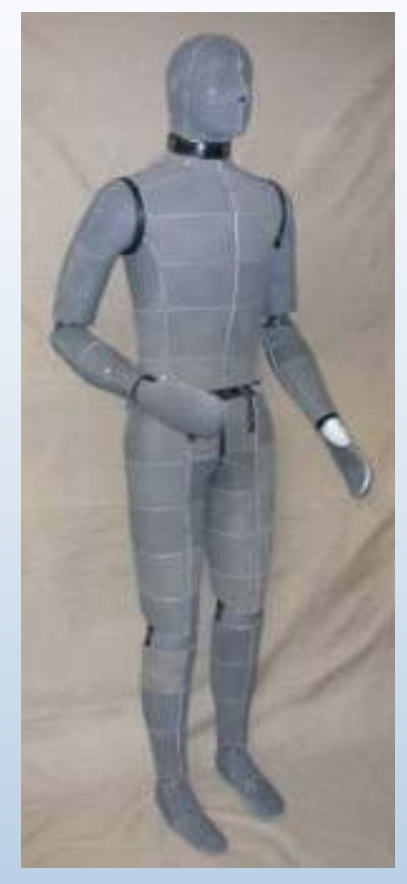

ADvanced Automotive Manikin

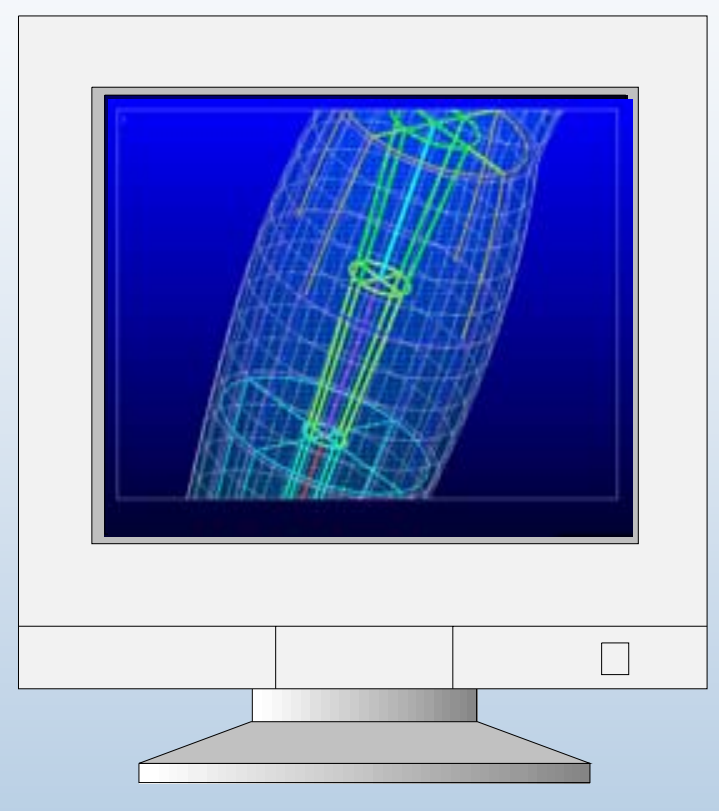

Human Thermal

Physiological Model

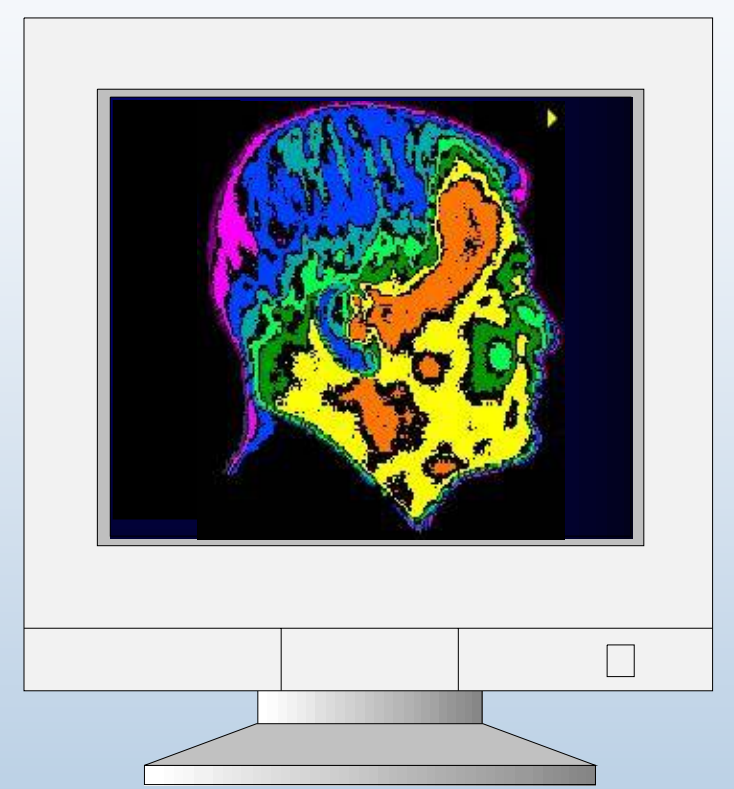

Human Thermal Comfort Empirical Model 


\section{ADvanced Automotive Manikin (ADAM)}

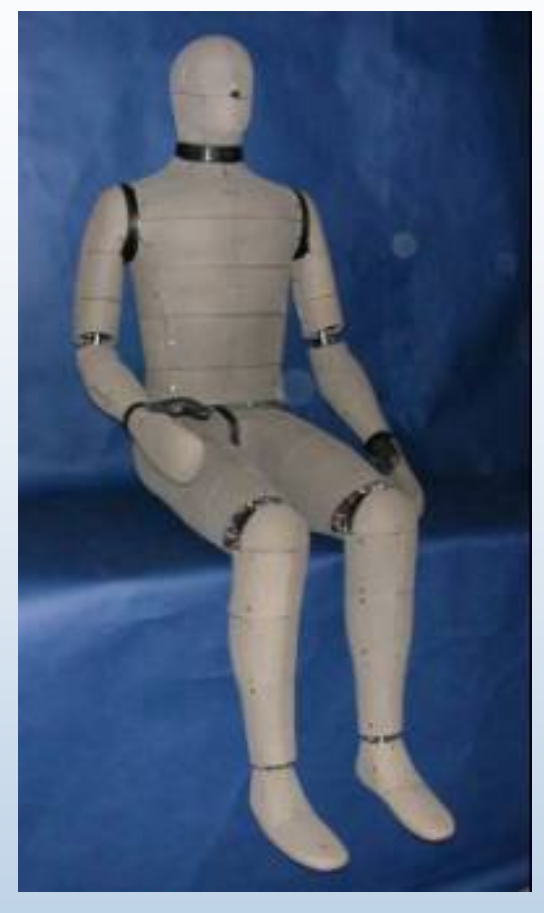

- On-board power, water, communications

- Designed to respond to a transient, nonuniform thermal environment like a human:

- Sweating

- Breathing

- Wears clothes

- $175 \mathrm{~cm}$ tall, $61 \mathrm{~kg}$

- 126 segments $\left(\sim 120 \mathrm{~cm}^{2}\right), 120$ zones
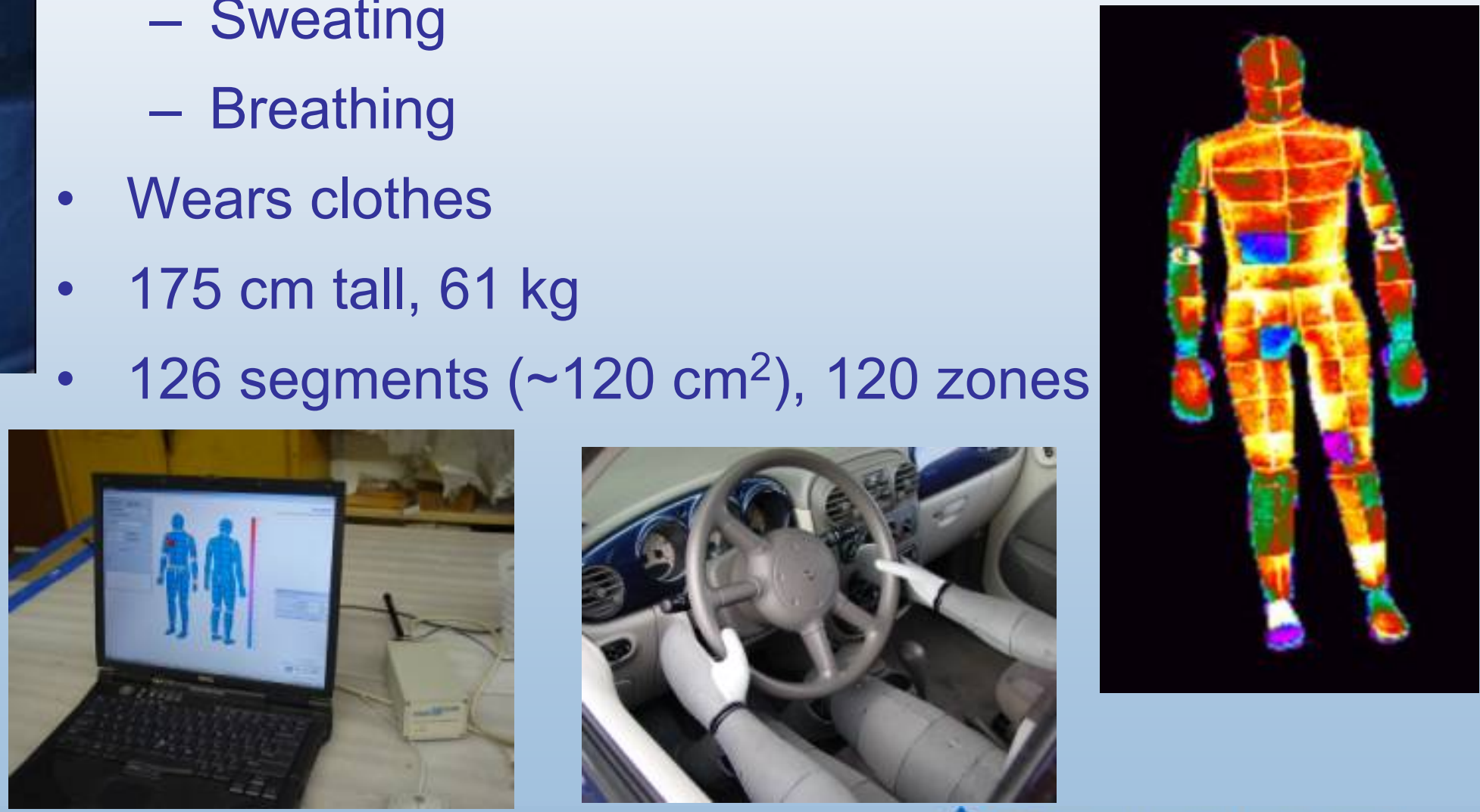


\section{Surface Segment Details}
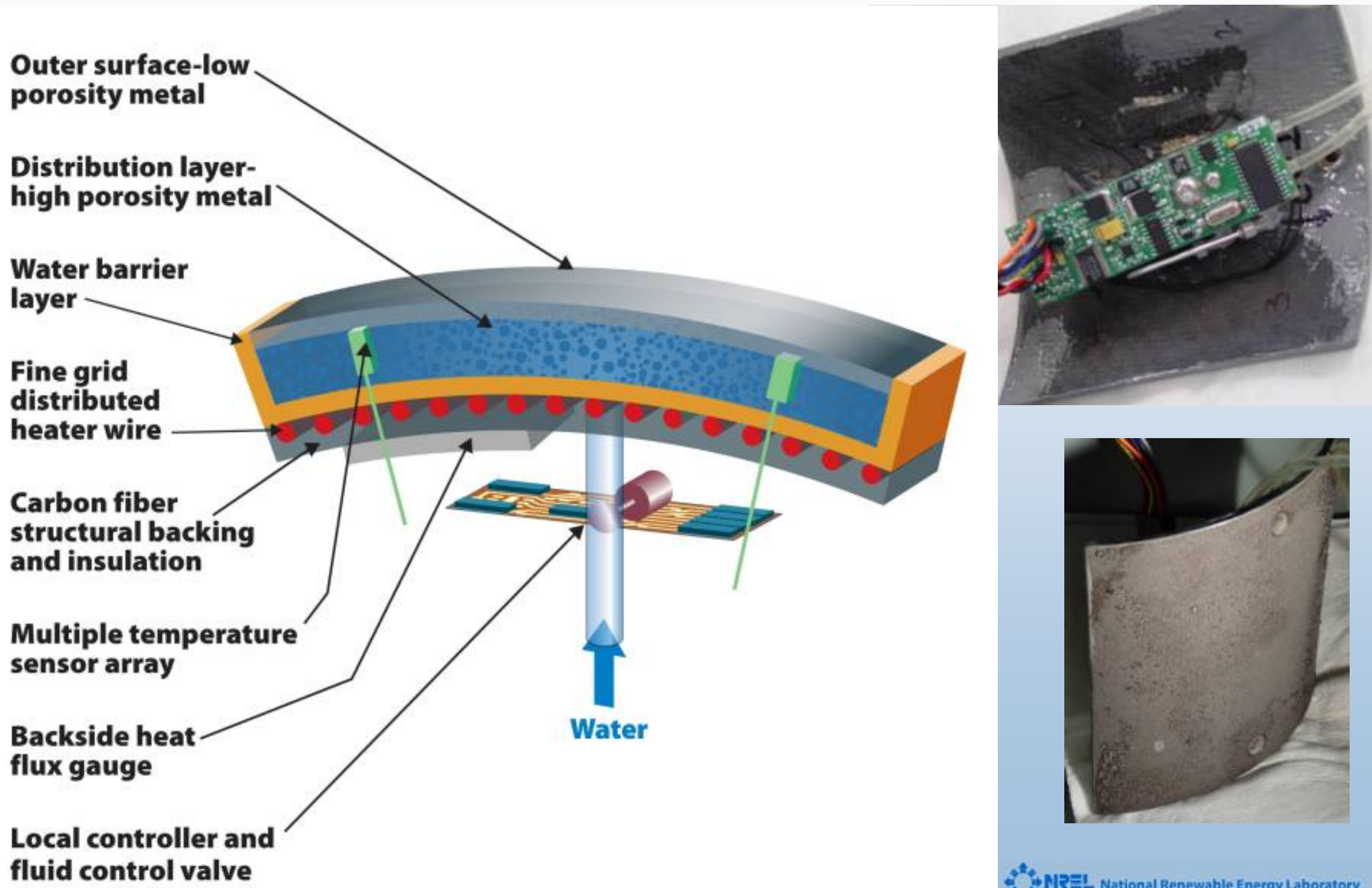

fluid control valve 


\section{ADAM's Carbon Fiber Skeletal System}
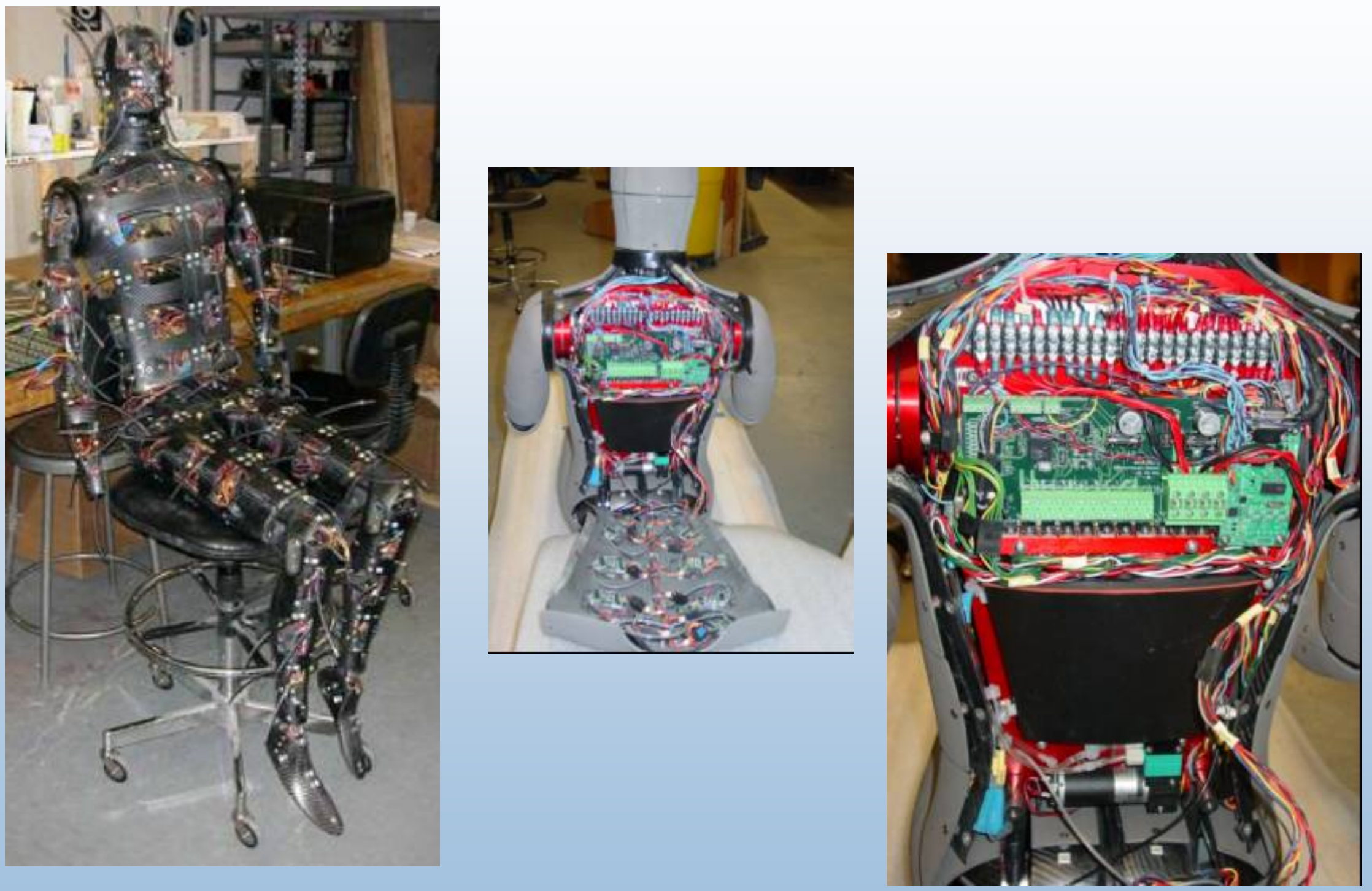


\section{Ready for a Brain!}
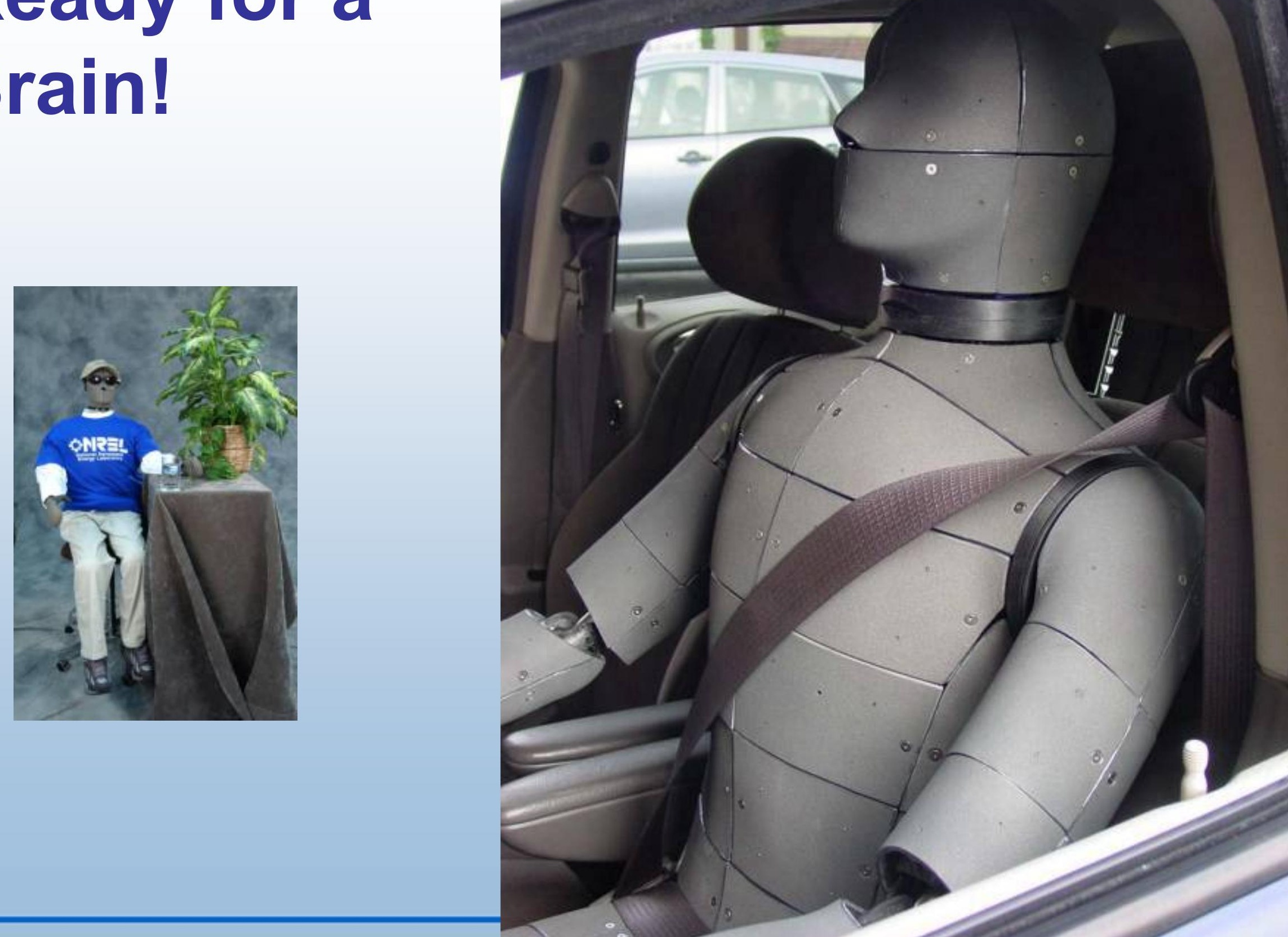


\section{Human Thermal Physiological Model Thermoregulatory System}

- Circulatory system (network of circular tubes of variable cross sections for arteries \& veins)

- Respiratory system (series of tubes between the mouth and lungs)

- Published control equations used for heat generation, sweat rate, shivering rate, vaso-dilation/constriction 


\section{Mesh}

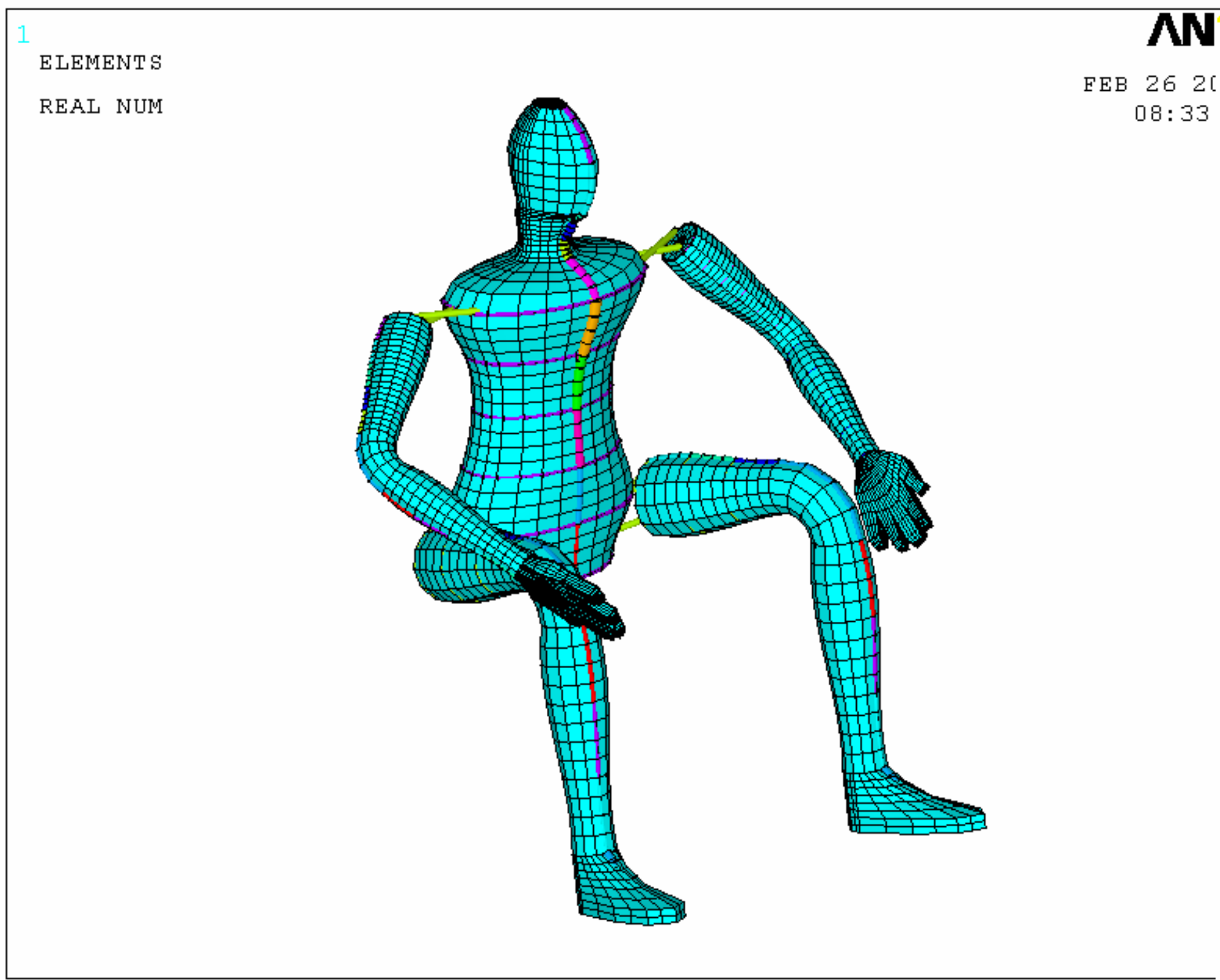




\section{Section View of Upper Right Leg}

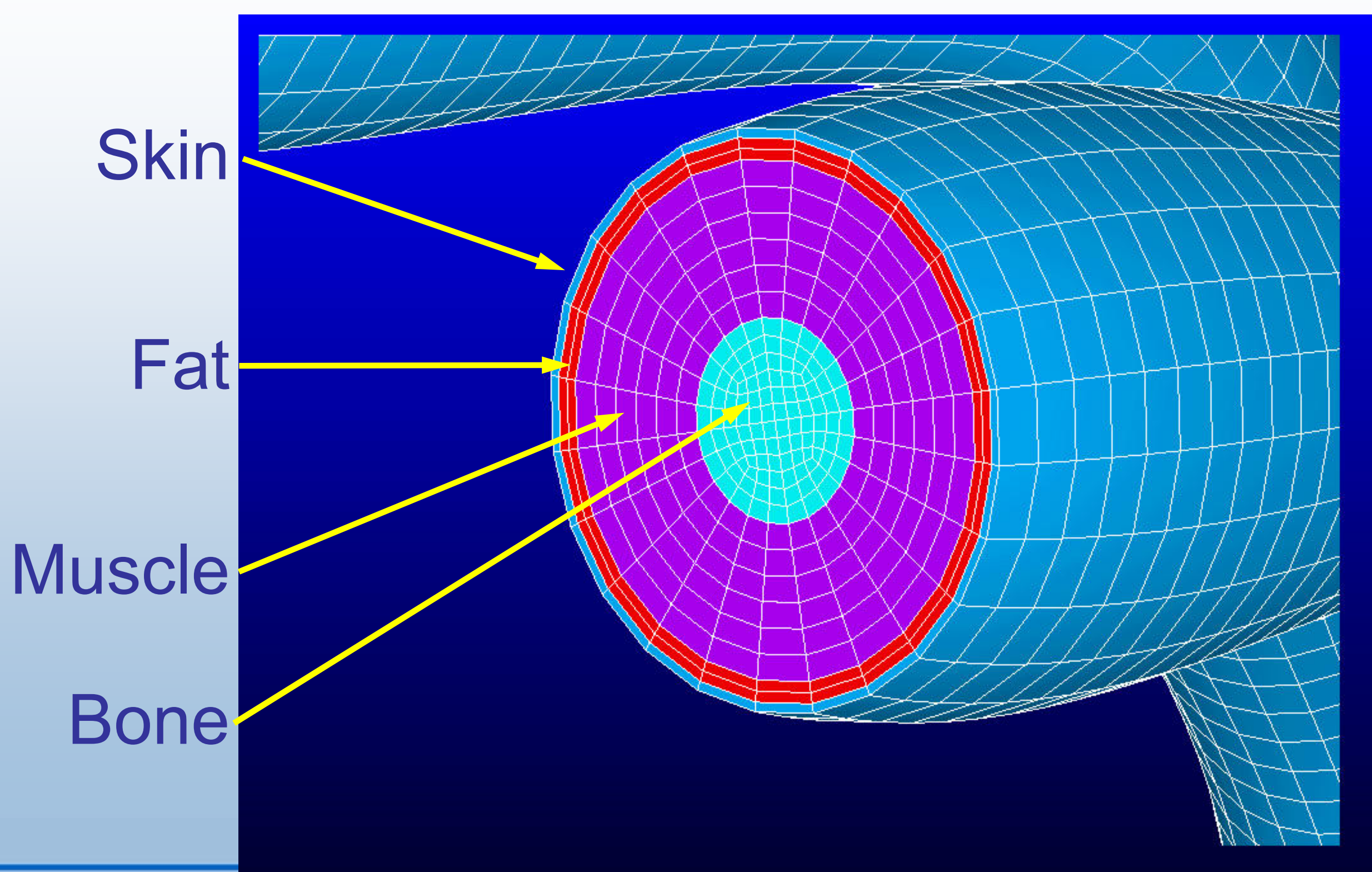




\section{Circulation Network}

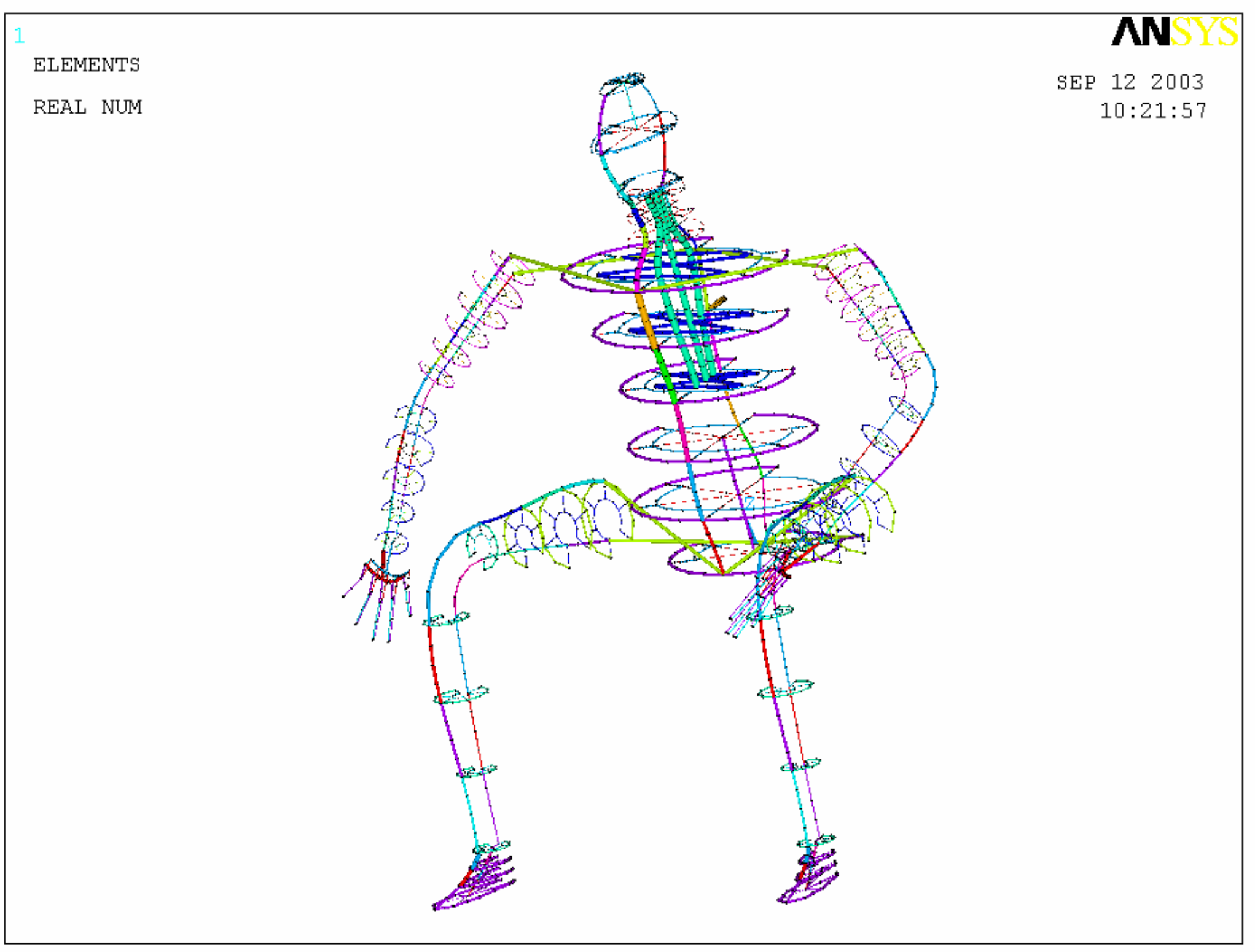




\section{Body Skin Temperatures}

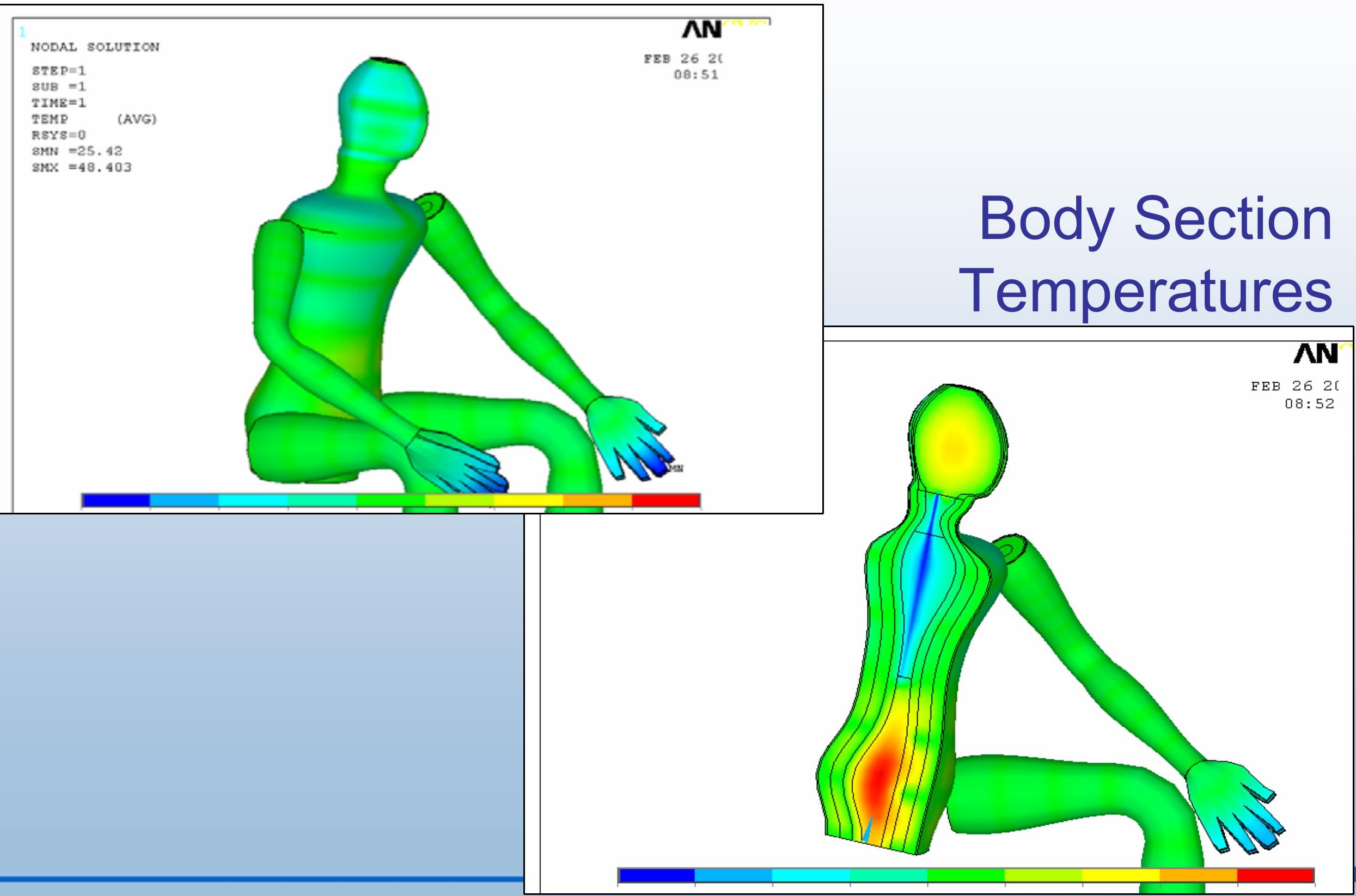




\section{The Human Thermal Comfort Empirical Model}

How You Feel Thermally

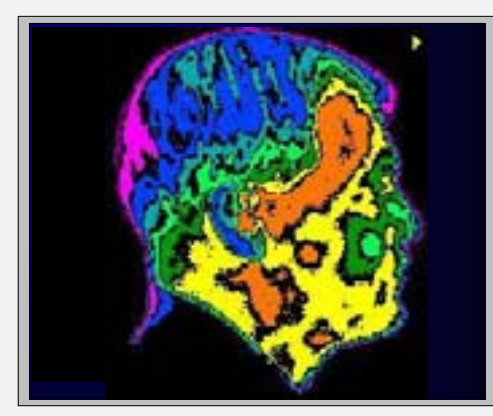

- Determines local thermal sensation

- Determines local and global thermal comfort (thermal perception) from local sensations

- Accounts for non-uniform and transient thermal environment of vehicle cabin
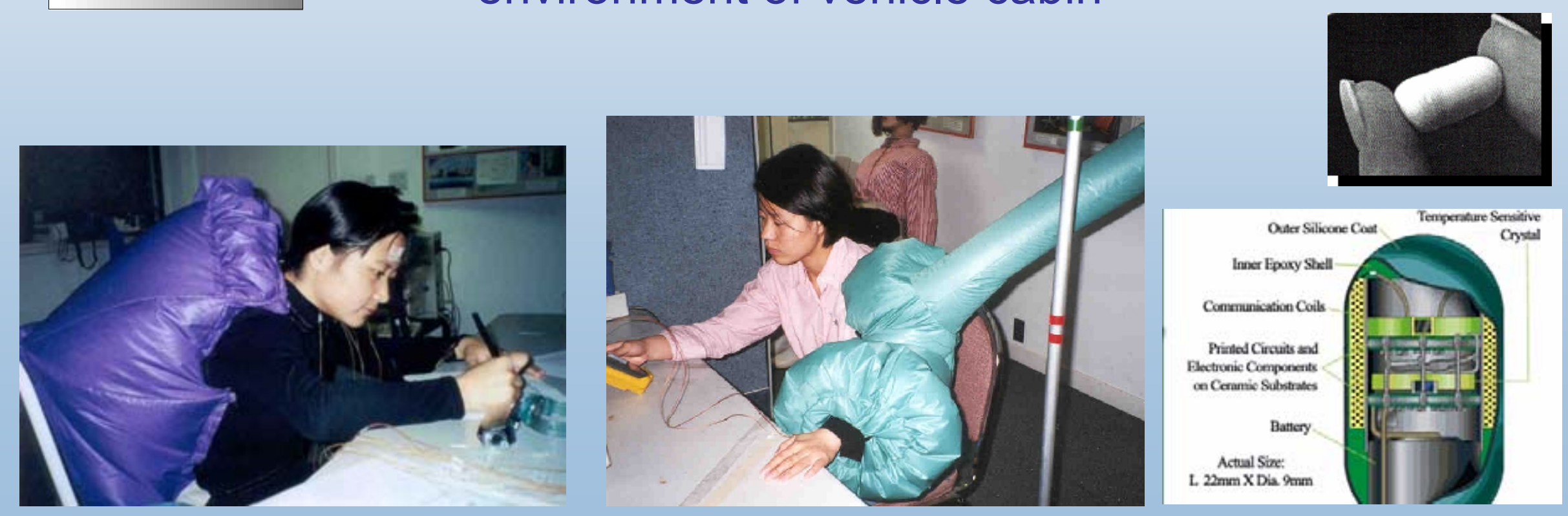


\section{Human Subject Testing}

- Individual segments (plus breathing temperature) were heated and cooled.

- 110 separate were tests performed at U.C. Berkeley.

- 64 tests were performed in Delphi wind tunnel.
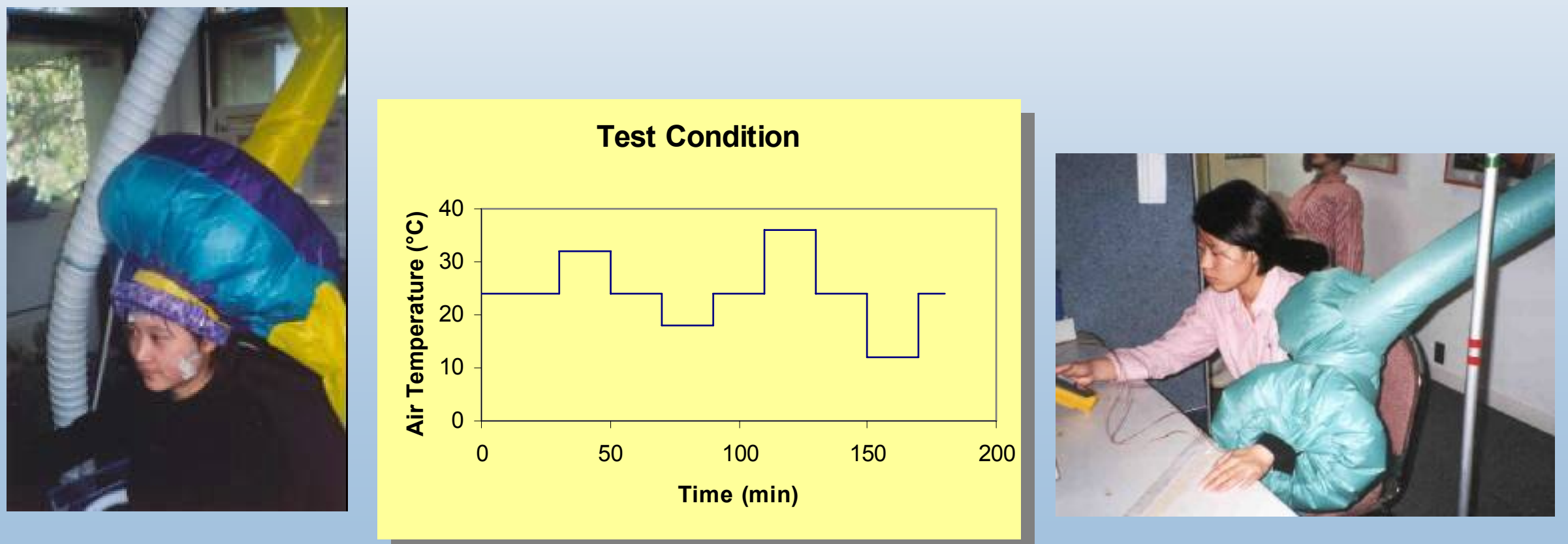


\section{Model Overview}

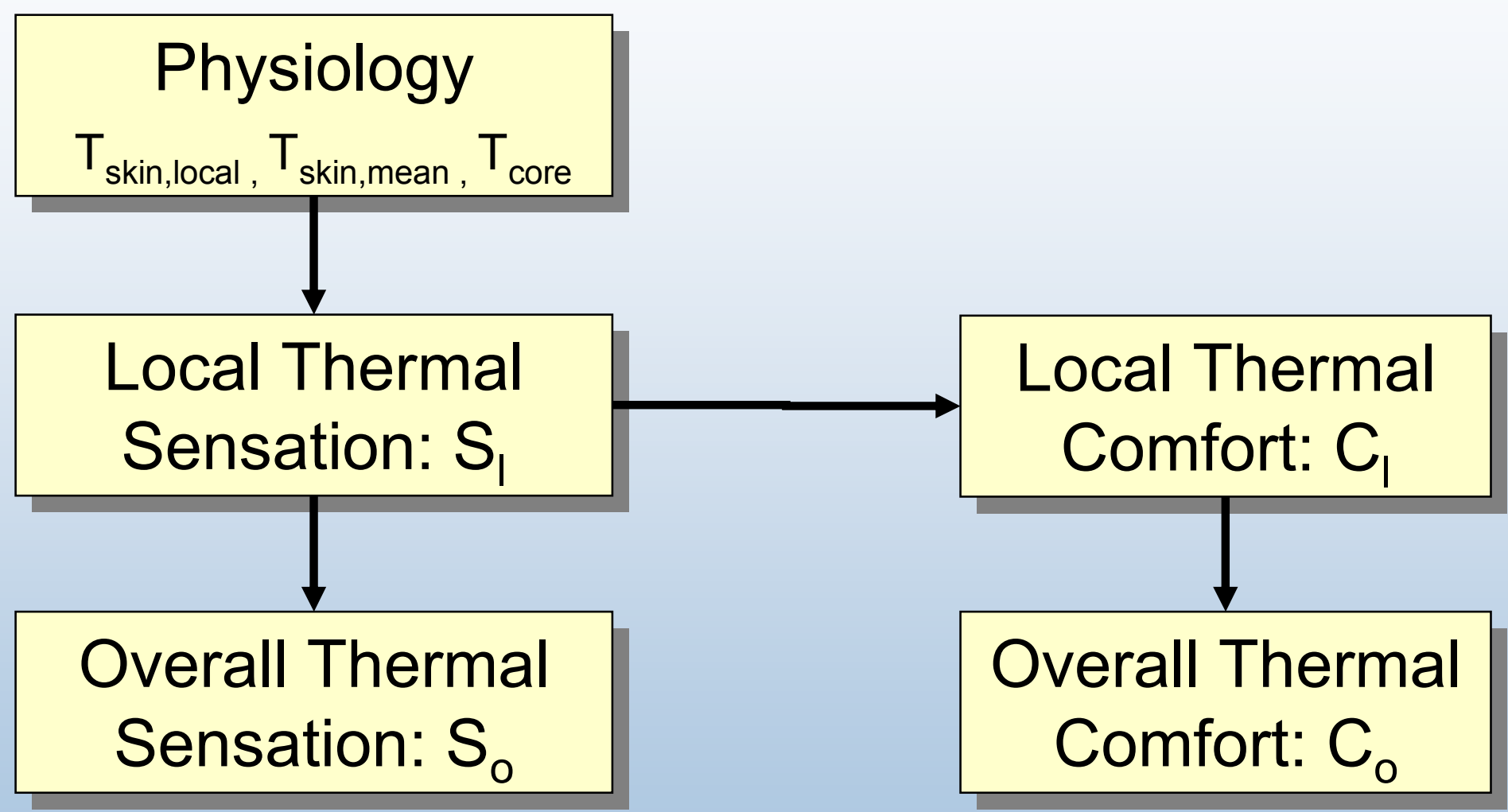




\section{Validation Test - UCB Data}

- Compare to skin temperature, sensation, and perception (comfort) data from literature

- ADAM in leotard

- Standing

- Single subject

- Tair $=28.6^{\circ} \mathrm{C}$

- Ref. Zhang (2004)

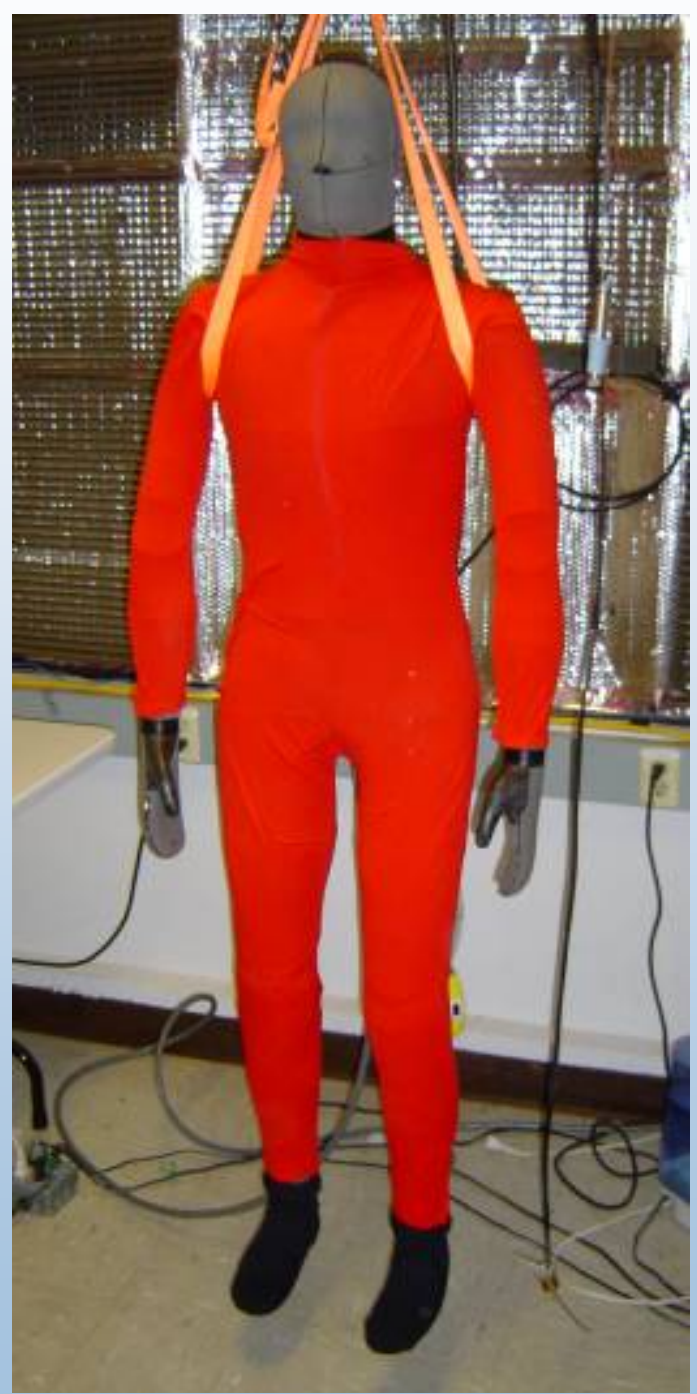




\section{Skin and Core Temperatures}

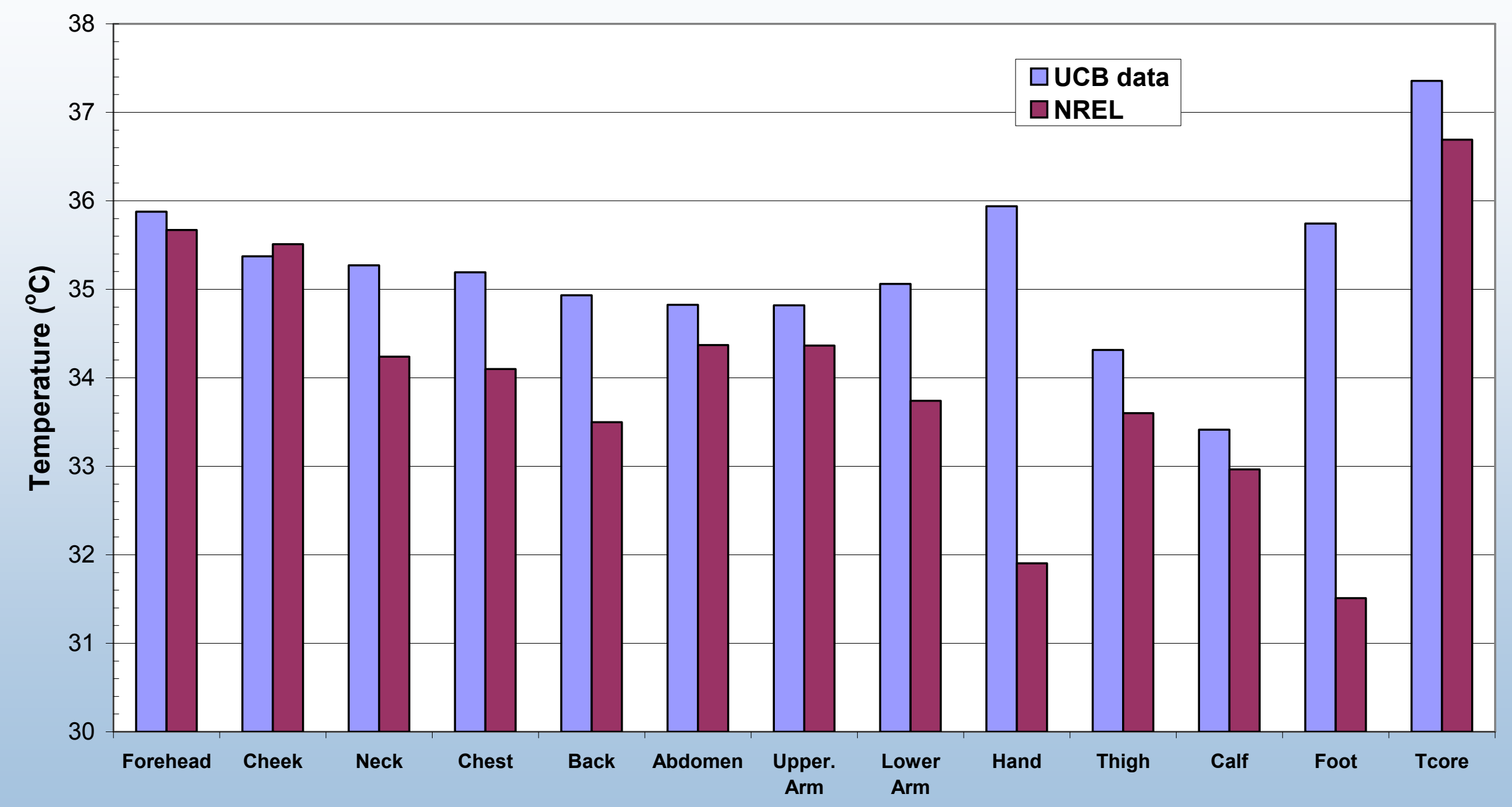




\section{Human \& Manikin Comparison}

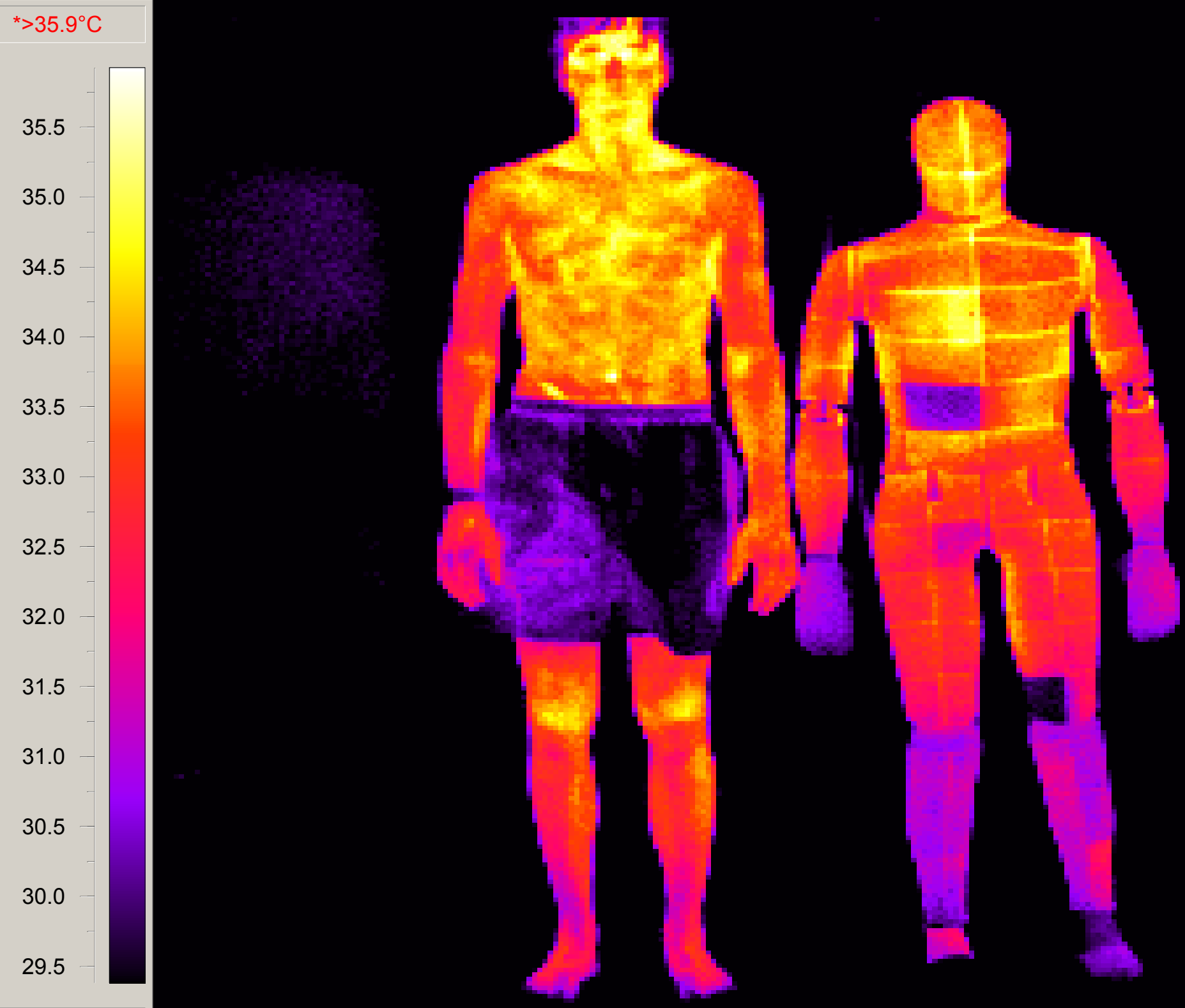


Linking the Physiological Model with a Swift Vehicle Model

CFD Model Enhancements:

- Sweat evaporation

- Clothing microenvironment

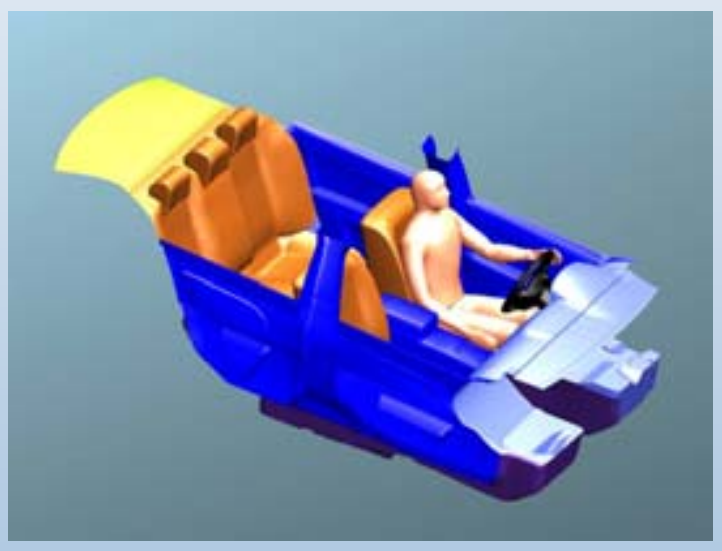

CFD simulates interaction with environment

\section{0 surface heat \\ fluxes transmitted}

Transmits 120 skin temperatures and sweat rates
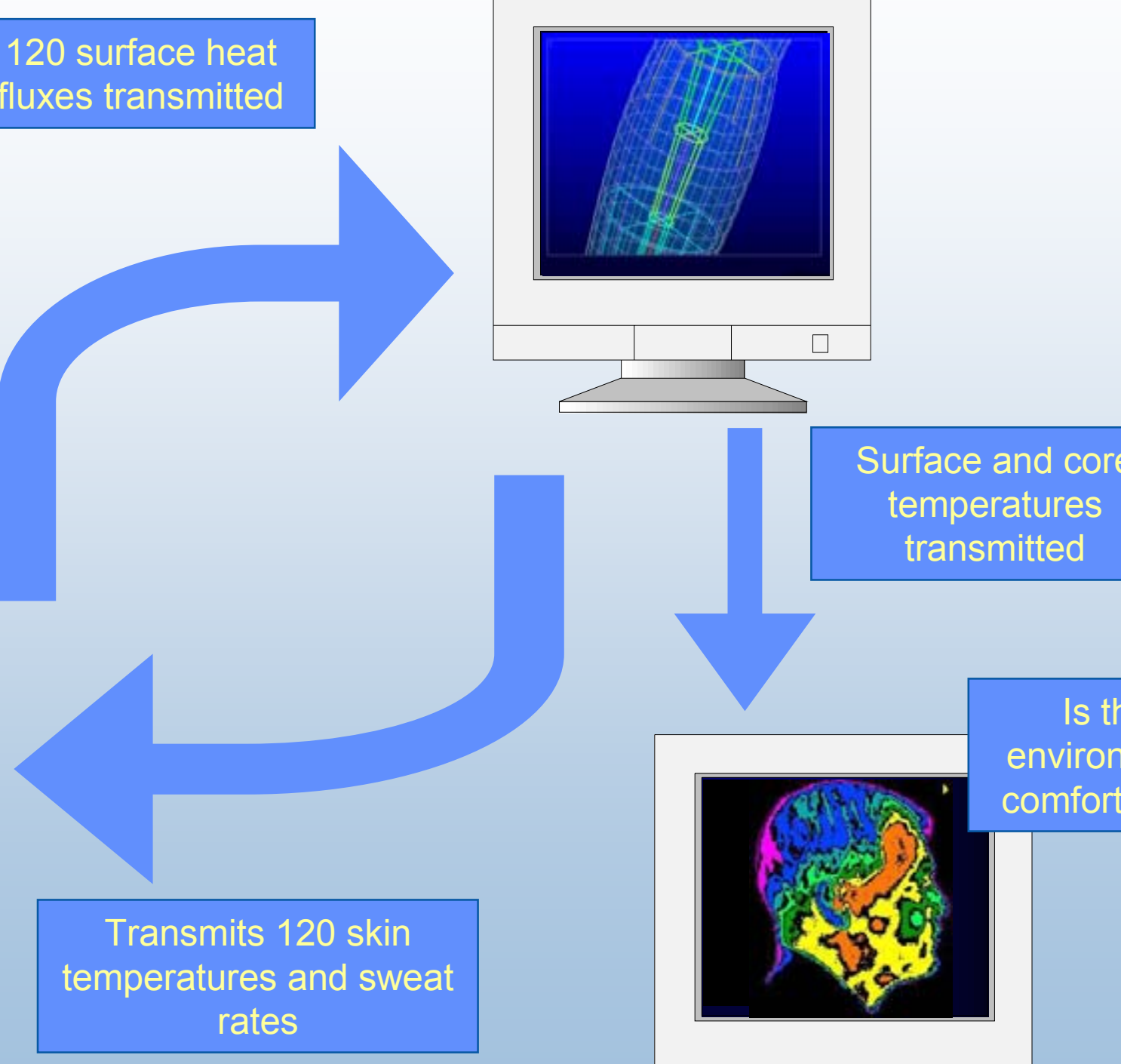

Is the environment comfortable? 


\section{Conclusions}

NREL has developed leading-edge cabin thermal management modeling tools in our effort to reduce vehicle fuel use

- Vehicle Solar Load Estimator

- Physiologically model with human-like skin temperature variations

- Thermal comfort model output using local thermal comfort sensitivity coefficients 


\section{Future Work}

- Continue validation testing

- Use validated manikin/model to assess advanced reduced fuel use HVAC concepts

- DOE is planning the $6^{\text {th }}$ International Manikin \& Modeling Meeting (6I3M)

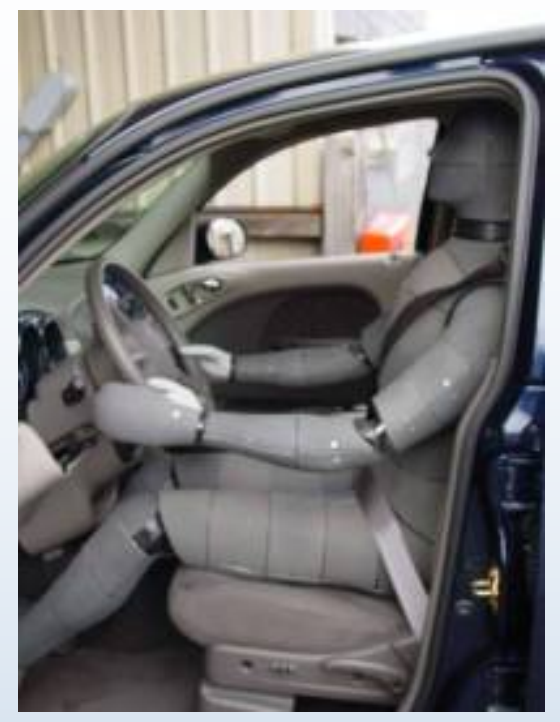

- April 2006

www.thermalmanikins.org

- Continue collaboration with AVL to assemble an improved cabin thermal management modeling process

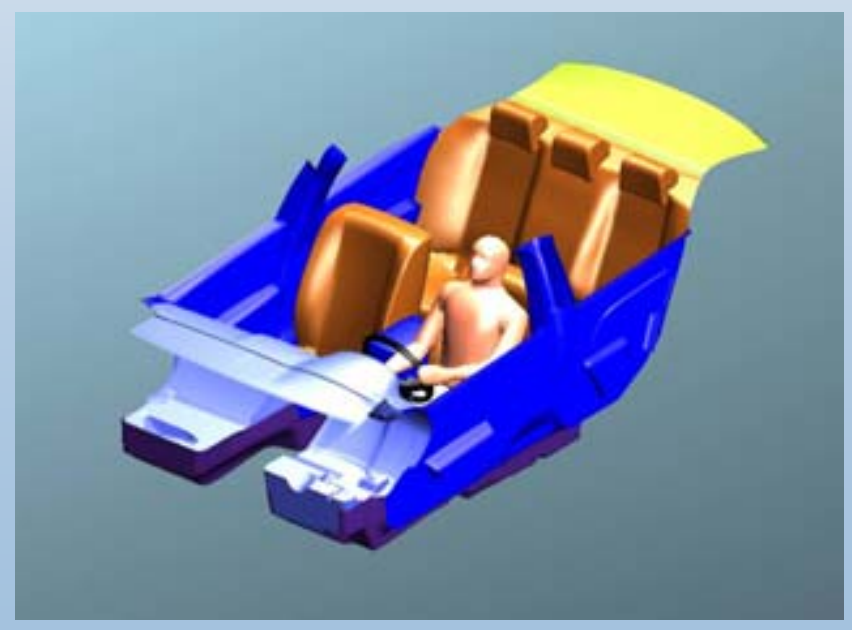




\section{Acknowledgments}

- U.S. Department of Energy

- Ed Wall, Office of FreedomCAR and Vehicle Technologies (OFCVT)

- Lee Slezak, OFCVT

- NREL

- Barbara Goodman, Director, NREL Center for Transportation Technologies and Systems

- Terry Penney, NREL OFCVT Technology Manager

- Rob Farrington, NREL Group Manager, Advanced Vehicle Systems

- Charlie King, NREL Technician

- AVL

- Reinhard Tatschl

- Wolfgang Baier

- Jurgen Wolfahrt (vif)

- University of California, Berkeley

- Charlie Huizenga

- Hui Zhang

- Measurement Technology Northwest - Rick Burke 


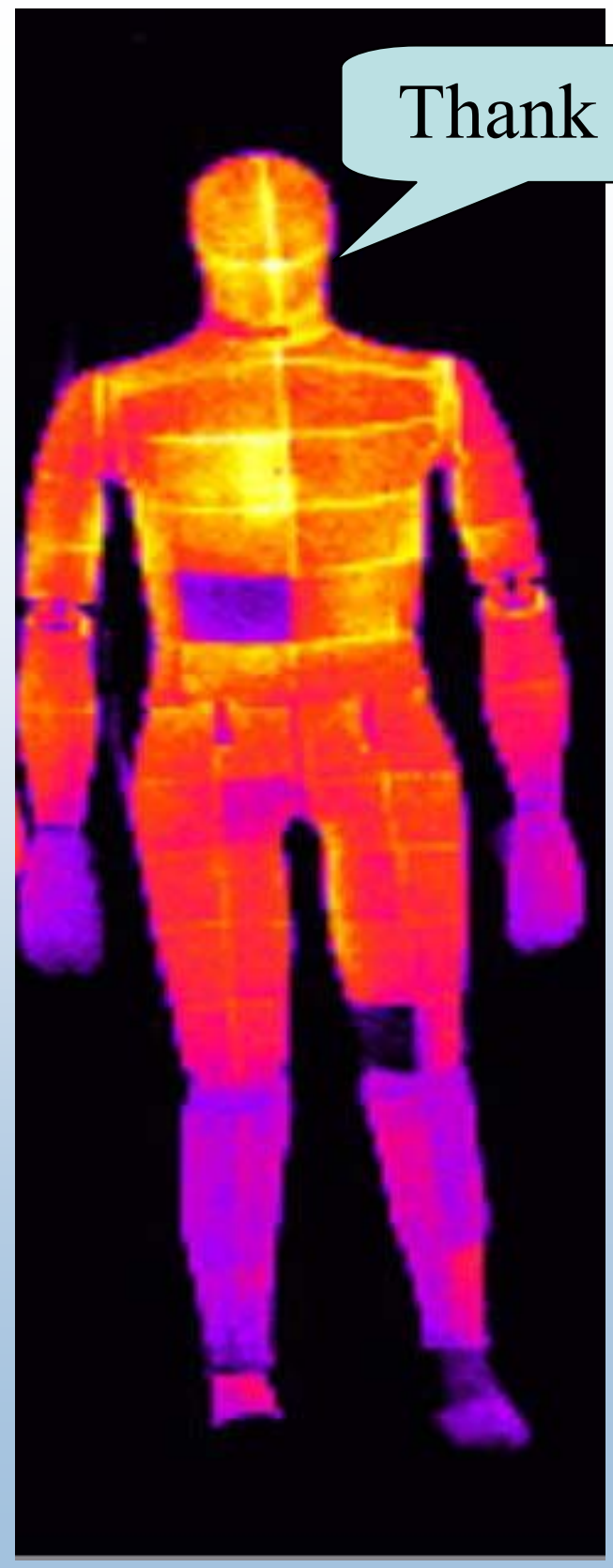

The U.S. Department of Energy's National Renewable Energy Laboratory
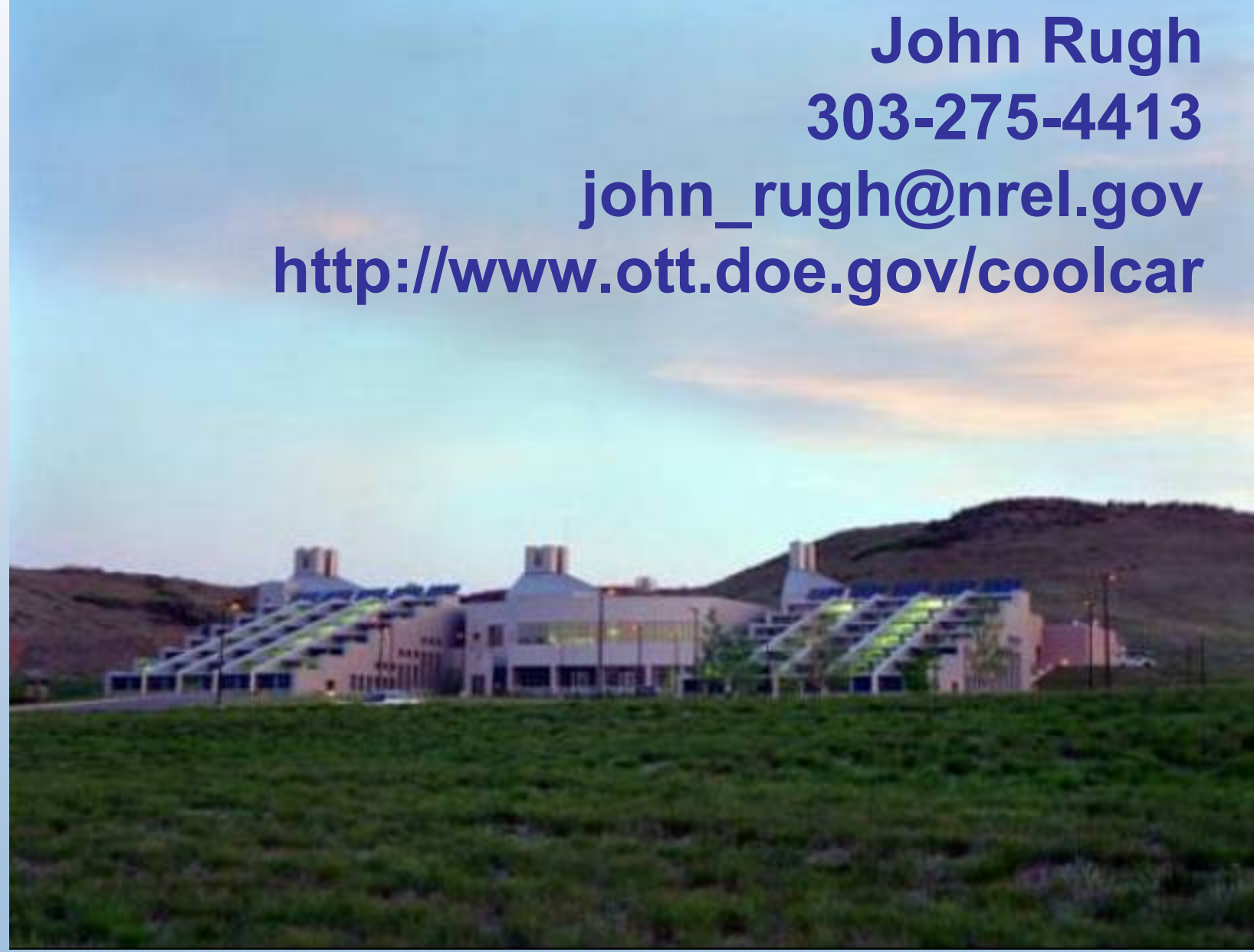